\title{
Controlling Organization and Forces in Active Matter Through Optically-Defined Boundaries
}

\author{
Tyler D. Ross ${ }^{1,2,5}$, Heun Jin Lee ${ }^{1,3}$, Zijie Qu ${ }^{1,2}$, Rachel A. Banks ${ }^{1,2}$, Rob \\ Phillips ${ }^{1,2,3,4}$, and Matt Thomson ${ }^{1,2,5}$ \\ ${ }^{1}$ California Institute of Technology, Pasadena, California, 91125, USA. \\ ${ }^{2}$ Division of Biology and Biological Engineering \\ ${ }^{3}$ Department of Applied Physics \\ ${ }^{4}$ Department of Physics \\ ${ }^{5}$ correspondence to: mthomson@caltech.edu, tross@caltech.edu
}

\begin{abstract}
A distinguishing feature of living matter is its ability to control force-generating structures that accomplish tasks such as chromosome segregation, intracellular transport, and cell movement. To generate and manipulate these micron-scale structures and force fields, cells create spatiotemporal patterns of molecular-scale activity. Here, we control micron-scale structures and fluid flow by manipulating molecular-scale protein activity set by dynamic optical boundaries in an engineered system of purified proteins. Our engineered system consists of microtubules and light-activatable motor proteins that crosslink and reorganize microtubules upon illumination. During reorganization, crosslinked motors do work to create non-equilibrium structures. We design light patterns that enable us to create, move, and merge microtubule asters with spatial and temporal precision. By composing these basic operations, we create microtubule networks that can span several hundred microns in length and contract at speeds up to an order of magnitude faster than the speed of an individual motor. By scaling the size and speed of contractile networks, we generate and sculpt advective fluid flows. Our work shows that dynamic boundaries enable control over active matter. The principles of boundarymediated control we uncover may be used to study emergent cellular structures and forces and to develop programmable active matter devices.
\end{abstract}

Living systems are capable of locomotion, reconfiguration, and replication. Underlying these phenomena are collections of force-generating, "active" molecules whose nanometer scale interactions are coordinated to form up to millimeter-scale structures and force fields [1-3]. A developing area of research is how the spatial and temporal patterning of these interactions generates forces and non-equilibrium structures that span orders of magnitude in scale. For example, experimental active matter systems of biological or synthetic molecules are capable of organizing into dynamic asters [4, 5] and spontaneously generating flows [6-9]. However, these experimental systems offer almost no spatial and temporal control, which restricts the study of their dynamics and limits their potential engineering applications.

Recent work has partially addressed these issues by using light to activate [10, 11] or localize [12, 13] active matter systems, but still lacks precise spatiotemporal control over organization and forces. In contrast, cells precisely control the organization of non-equilibrium structures like their spindles and lamellipodia by spatiotemporally modulating the localization and activity of proteins [14]. Here, 
we control the non-equilibrium organization of microtubule filaments by defining motor protein activity in space and time with dynamic, optical compartments. With our engineered system, we develop basic operations to create, move, and merge microtubule structures. By combining these operations, we construct a set of rules that provide a framework for studying and manipulating active matter, thereby enabling us to discover new phenomena and their underlying principles.

Our scheme is based on a well-studied active system composed of stabilized microtubule filaments and kinesin motor proteins [4-8, 15-17]. In the original biochemical system, kinesin motors are linked together by biotin-streptavidin bonds. Linked kinesin motors bind to adjacent microtubules, which leads to the crosslinking of microtubules. As linked microtubules pull on motors, a variety of phases and structures spontaneously emerge, such as asters, vortices, and networks. However, spatial and temporal control of these structures is limited [5, 12].

We re-engineered the motor-microtubule system so that light activates the linking of motors (Fig. 1A). Descriptions of all materials, methods, data analysis and modeling are detailed in the Supplementary Information section. Light patterns are projected into the sample throughout its depth and determine when and where motors will crosslink microtubules. Outside of the light excitation volume, microtubules remain disordered, while inside the light volume, microtubules bundle and organize. For a cylinder pattern of light excitation, microtubules organize into a 3D aster (Fig. 1B). Here, the cylinder pattern acts as an operation for creating aster structures.

Our temporal control over aster formation allows us to study the dynamics of their creation and decay (Fig. 1C) (Video 1) through time lapse imaging (SI 2.1). We characterize these dynamics by measuring the standard deviation of the spatial distribution of intensity of fluorescent-labeled microtubules (SI 2.5). During aster formation, we find that, after a delay phase of $\sim 1 \mathrm{~min}$, the distribution of microtubules within a cylinder pattern contracts. After 10-15 min, we observe that the spatial distribution reaches a steady state, indicating the aster is fully formed. We measure the aster size at $15 \mathrm{~min}$ by image segmentation (SI 2.2). Once the excitation light is removed, asters begin to decay into free microtubules. The spatial distribution of microtubules widens over time, returning to the initial uniform distribution. Aster decay is reversible, as demonstrated by reforming of an aster in the same location (Fig. S6). Thus, we use the timing of a cylinder of light as an operator to control the dynamics of asters.

We investigate the scaling properties of asters by varying the diameter of the excitation cylinder. During aster formation, the spatial distribution of microtubules contracts linearly with time (SI 2.6.2), which we characterize by a contraction rate. The contraction rate grows linearly with the diameter of the excitation cylinder (Fig. 1D). Similar behavior has been observed for contractile actin networks [18]. During aster decay, the unbound microtubules appear to move in a diffusive manner (SI 2.6.3), which we characterize by an effective diffusion coefficient. This diffusion coefficient is independent of aster size (Fig. 1E) and is in rough agreement with what is expected for freely diffusing microtubules (SI 2.3). We also control the size of an aster by varying the excitation cylinder diameter. We find that aster size scales with excitation diameter to the $2 / 3$ power (Fig. 1F). This dependency is expected based on the scaling of the reaction volume with excitation diameter (SI 2.4). The aster scaling behavior shows similarities to the dependence of spindle size on confining volumes [19]. We note that for excitation cylinders with diameters less than $25 \mu \mathrm{m}$, we are not able to form clearly resolved asters. These results demonstrate how light can create boundaries of protein activity, similar to how cells control dynamic structures through patterned molecular activity.

We dynamically position light patterns and asters relative to the sample slide by moving the mi- 
(A)

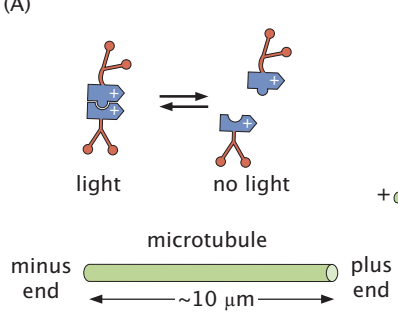

(C)
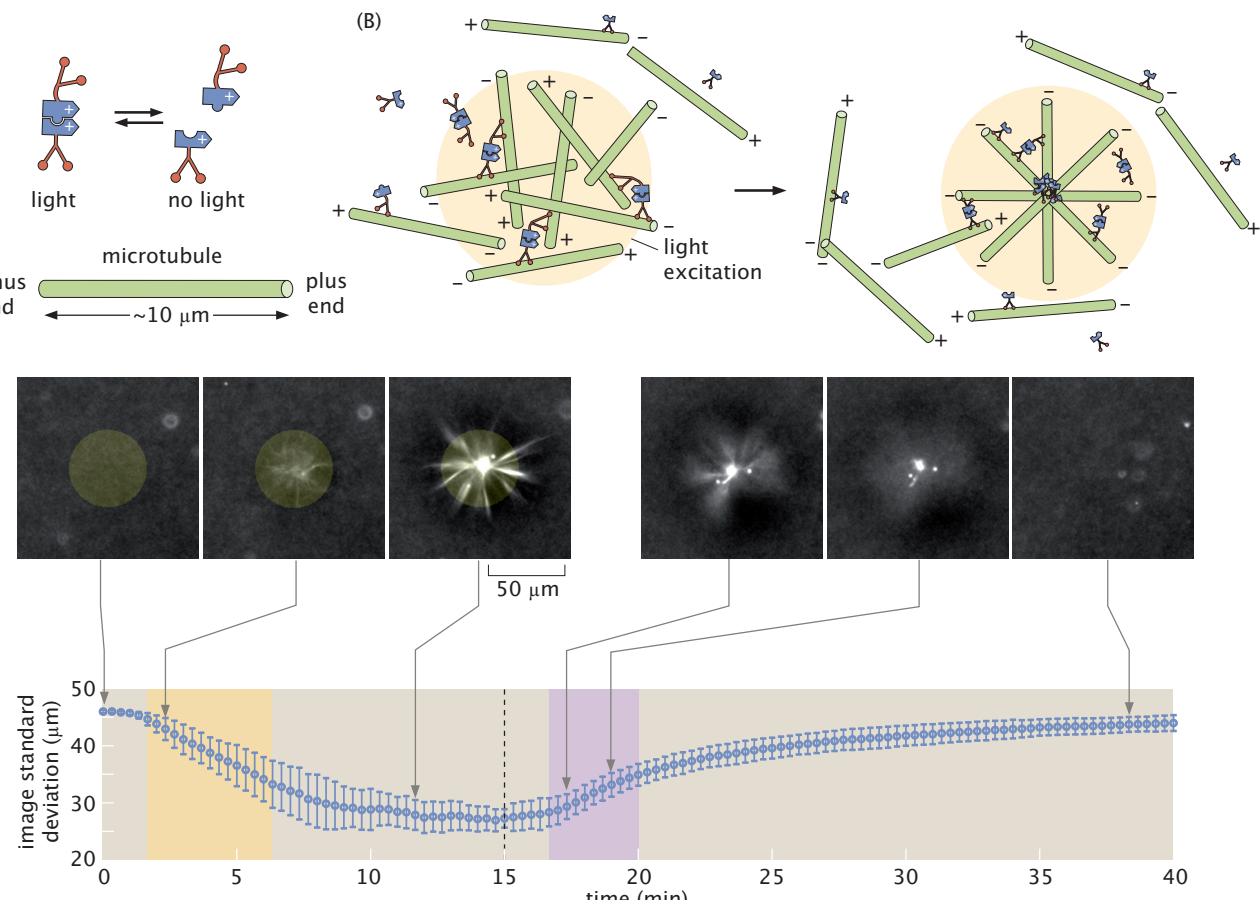

(D)

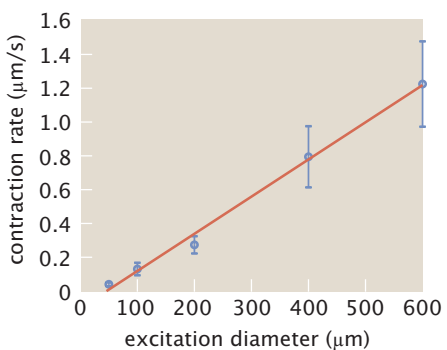

(E)

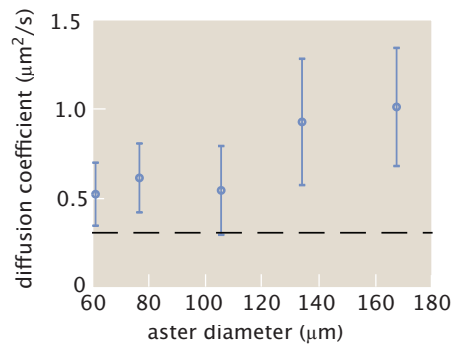

(F)

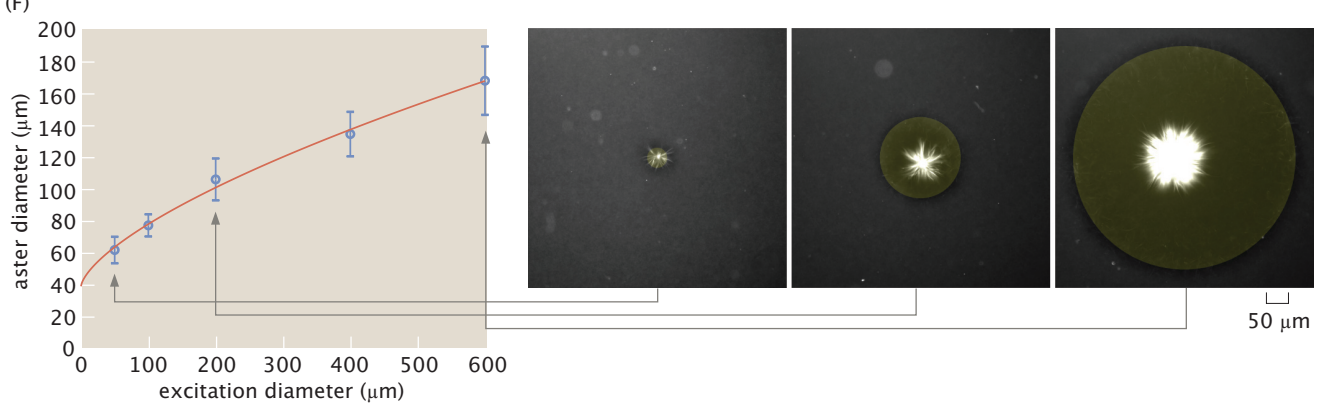

Figure 1: Light-switchable active matter system enables optical control over aster formation, decay and size. (A) Schematic of light-dimerizable motors. (B) Schematic of light-controlled reorganization of microtubules into an aster. (C) Images of labeled microtubules during aster assembly and decay and corresponding image spatial standard deviation versus time. The dashed line is when the activation light is removed, transitioning from creation to decay. The orange region shows the time interval used to find the contraction rate. The lavender region shows the time interval used to find diffusion coefficients. (D) Contraction rate versus excitation diameter. The red line is a linear fit. (E) Diffusion coefficients versus aster diameter. The dashed line represents the diffusion coefficient of a $6 \mu \mathrm{m}$ microtubule (SI 2.3). (F) Aster diameter versus excitation diameter with representative images. The red curve is a fit to a $2 / 3$ power law expected by scaling arguments. In panels $\mathrm{C}, \mathrm{D}$, $\mathrm{E}$, and $\mathrm{F}$ the data points represent the mean of 5 experiments and the error bars are the associated standard deviations. In panels $\mathrm{C}$ and $\mathrm{F}$, the yellow shaded disks represent the light pattern. 
(A)

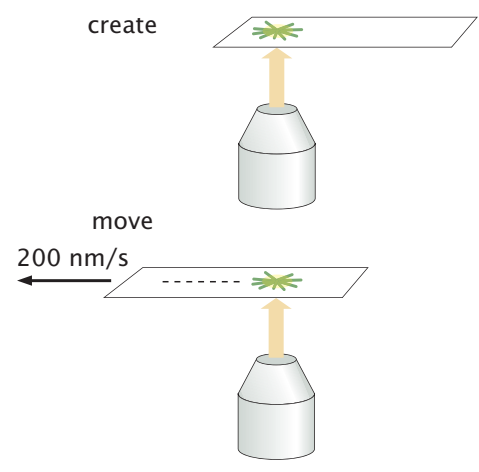

(C)

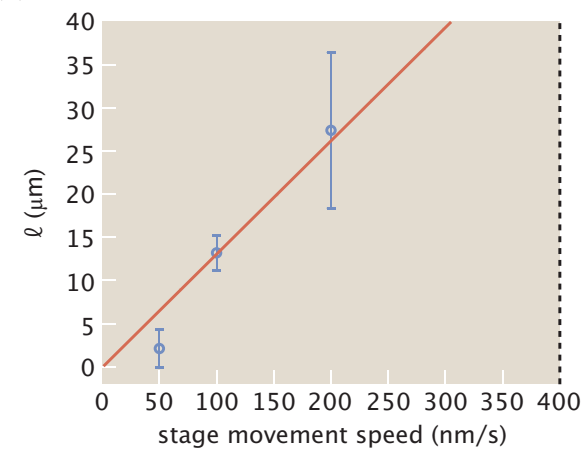

(E)

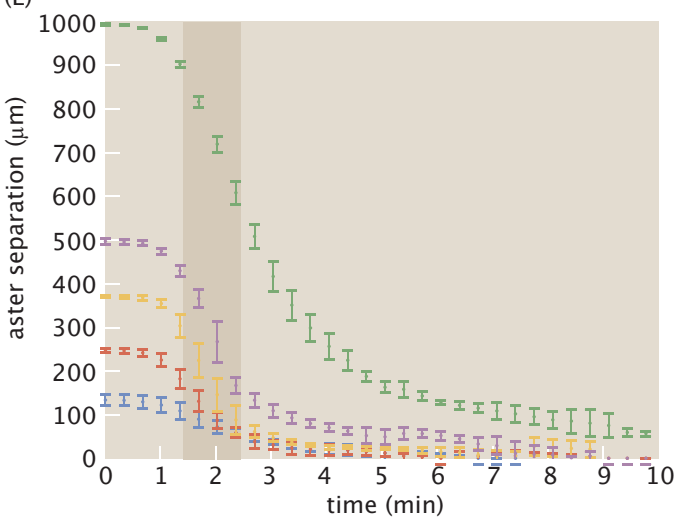

(B)

(D)
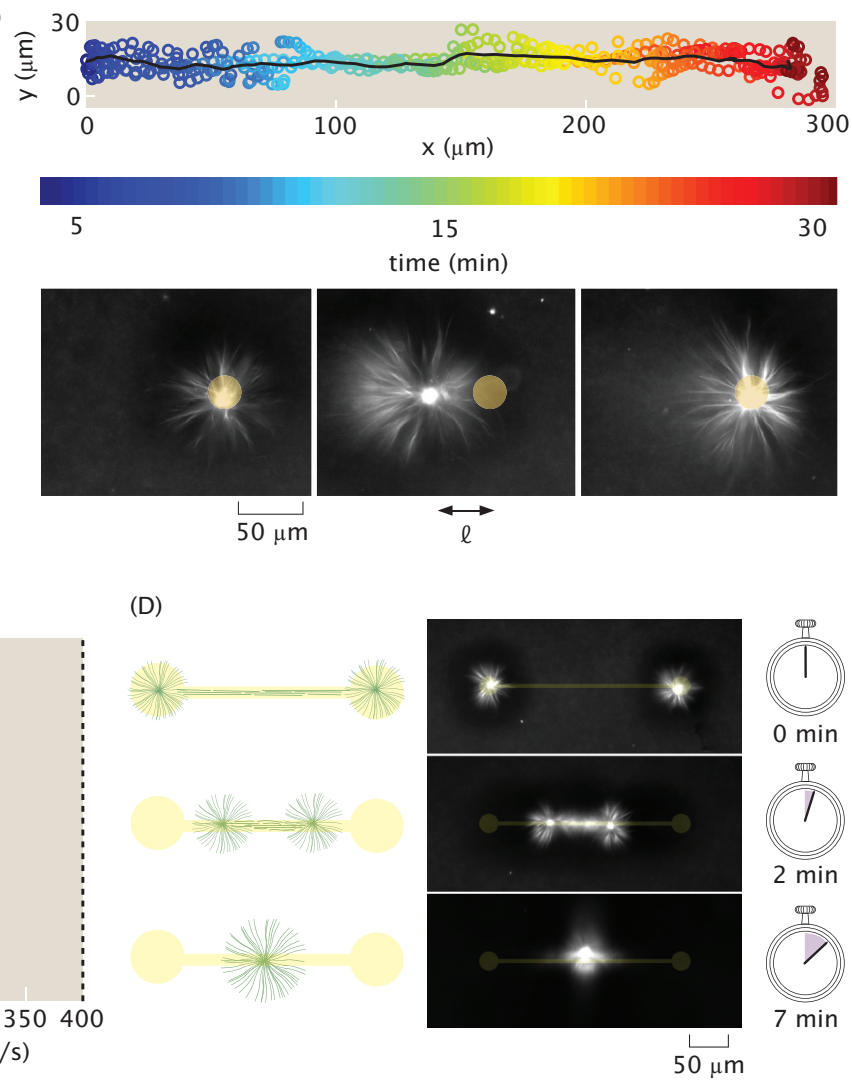

(F)

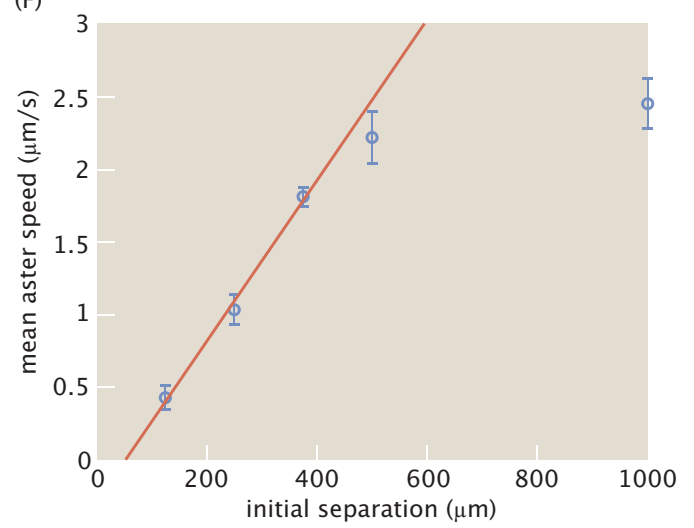

Figure 2: Moving and merging operations of asters with dynamic light patterns. (A) Asters are moved relative to the slide by repositioning the microscope stage. (B) Overlay of 2D trajectories of aster movement relative to slide moving at $200 \mathrm{~nm} / \mathrm{s}$. The line represents the mean trajectory. Time lapse images show the position of the aster relative to the light pattern. $\ell$ is the displacement of the aster from center of the light pattern. (C) $\ell$ versus stage speed. The dotted line at 400 $\mathrm{nm} / \mathrm{s}$ represents the escape velocity. The red line is a linear fit. (D) Illustration of the aster merge operation by a connected excitation pattern and the corresponding time series of images. (E) Distance between merging asters over time for different initial separations. (F) Speeds of aster mergers in the shaded region of panel E. The red line is a linear fit to the first three data points. In panels $\mathrm{B}, \mathrm{C}, \mathrm{E}$, and $\mathrm{F}$ the data points represent the mean of 5 experiments and the error bars are the associated standard deviations. 
croscope stage (Fig. 2A). We are also able to move the aster by directly moving the light pattern, however, moving the stage allows for a greater range of travel. As the stage moves, the asters track with the light pattern, traveling up to hundreds of microns relative to the slide (Fig. 2B) (Video 2). The aster maintains a steady state distance $\ell$ between itself and the light pattern (Fig. 2C). Based on the reorientation of the aster arms, the aster appears to be pulled in tow behind the light pattern, perhaps by the aster arms or newly-formed microtubule bundles in the light pattern. We find that asters are always able to track the pattern for stage speeds up to $200 \mathrm{~nm} / \mathrm{s}$. At $400 \mathrm{~nm} / \mathrm{s}$, asters are not able to stay with the pattern. Based on these observations, we define an "escape velocity" that occurs between 200-400 nm/s, which sets the limit of how fast we can move asters relative to the sample slide. This speed is comparable to the motor speeds measured in gliding assays (Fig. S7). When the stage stops moving, the aster returns to the center of the light pattern, indicating that the aster is experiencing a restoring force. These observations are consistent with the notion that the moving aster is in an effective finite potential (SI 2.9).

We find that asters can be combined together when connected with patterned light, leading to an aster merger operation (Fig. 2D) (Video 3). At the beginning of the merging process, we observe that a network of bundled microtubules forms, which connects the asters. After a lag time similar to that observed for aster formation, the connecting network begins to contract and the asters move towards each other (Fig. 2E). The speed at which asters merge is found to increase as a function of linking distance up to a speed of roughly $2.5 \mu \mathrm{m} / \mathrm{s}$ (Fig. 2F). For initial separations up to 500 $\mu \mathrm{m}$, merger speeds scale linearly with separation. The scaling of aster merger speed as a function of distance is similar to the observed relationship of contraction rate as a function of the excitation cylinder size discussed above. We note that the maximum observed merger speed is about an order of magnitude higher than the speeds observed during gliding assays (Fig. S7), which is analogous to how cell migration speeds can exceed single motor speeds [20].

Performing successive operations remains a fundamental step towards engineering with active matter. Our ability to form dynamic light-defined compartments of active molecules enables us to execute multiple aster operations. By composing aster creation operations, we are able to form asters of differing sizes and place them at prescribed positions in parallel (Fig. 3A-B) (Video 4). Once asters are created they can be simultaneously moved by using multiple dynamic light patterns (Fig. 3C-D) (Video 5). Further, aster trajectories are not limited to rectilinear motion but can be moved along complex trajectories (Fig. 3E-F) (Video 6). Since the aster lags behind the light pattern and fluctuates along its trajectory, we expect these effects to limit the fidelity of programmed trajectories. Further, we observe that there is an inflow of microtubule bundles that are created in the light pattern, which feeds into the lagging aster, as we found in Fig. 2B. From the trajectory images (Fig. 3D, F), we see that there are also outflows of microtubules, which appears as "comettail" streams following the asters. These mass flows illustrate some of the complex non-equilibrium dynamics that are introduced by moving boundaries of molecular activity.

In our aster merging, moving, and trajectory experiments, we observed fluid flow of the buffer, as inferred by the advection of microtubules and small fluorescent aggregates. Similar cytoskeletal-driven flow is critical for the development and morphogenesis of various organisms including many plant species [21-26]. Based on these observations, we sought to construct a set of rules for generating and tuning flows. We can generate fluid flows of the background buffer directly by projecting only the rectangular bar pattern used during aster linking (Fig. 4A) (Video 7). Fluid flows are measured by tracking inert tracer particles in brightfield. The brightfield images reveal the formation of a dense microtubule network which undergoes structural changes (Fig. 4B) (Video 8). The resulting flow field is a distinct spatial pattern (Fig. 4C) and is stable for the duration of the experiment 

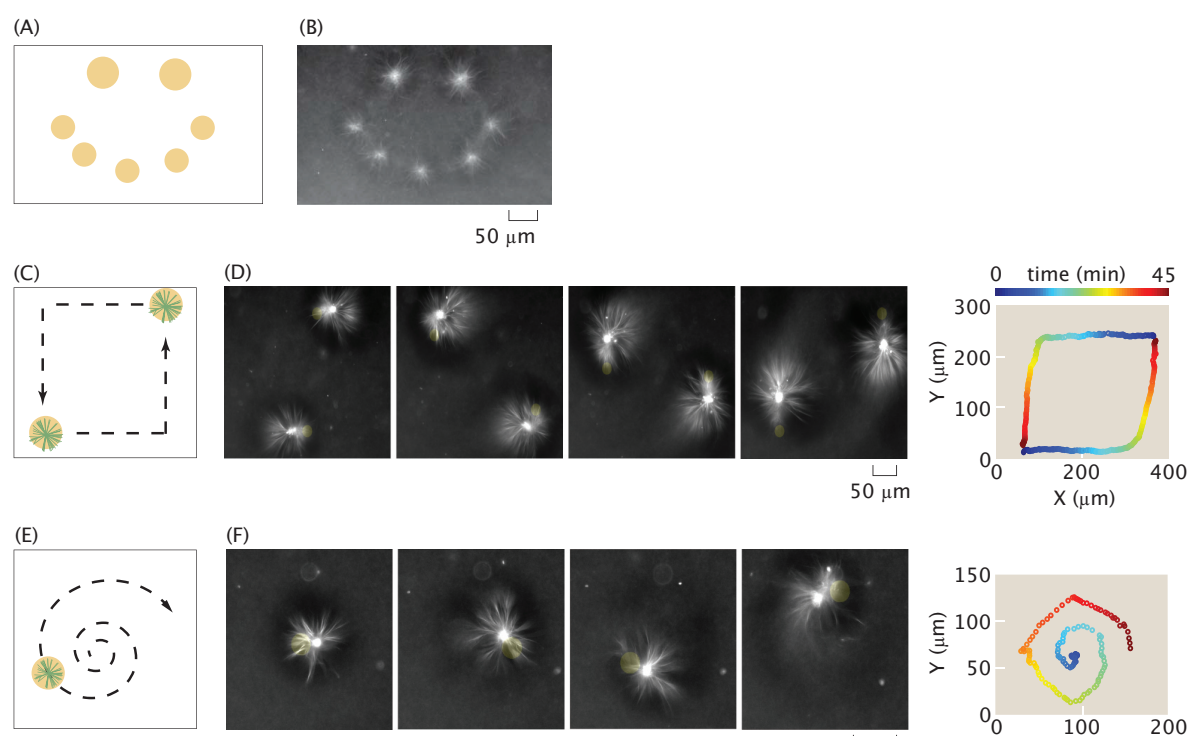

(F)
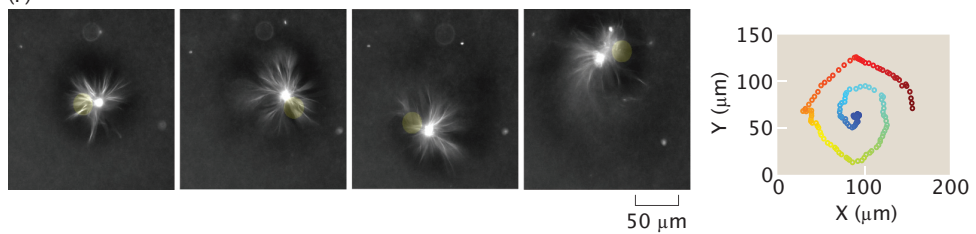

Figure 3: Operations for creating and moving asters are composed to make different desired patterns and trajectories. (A) Sketch for using excitation cylinders to simultaneously pattern asters of different sizes. (B) Resultant pattern of asters corresponding to A. (C) Illustration of simultaneous control of two different aster trajectories, as indicated by the dashed arrows. (D) Time lapse and the $2 \mathrm{D}$ trace of the aster trajectories corresponding to $\mathrm{C}$. The trajectory trace is color-coded to represent progression in time. (E) Dynamically projected spiral to illustrate curvilinear motion. (F) time lapse and the 2D trace of the aster trajectory. Time is color coded as in D.

\section{(SI 2.11).}

We are able to manipulate the properties of the flow field through the geometry of the activation volume. By varying the length of the rectangular bar, we observe that both the speed and size (SI 2.13) of the flow field increase linearly with the bar length (Fig. 4D-E). The speed scaling is similar to the observed relationships of aster merging speed versus separation and formation rate versus excitation diameter. By superimposing activation bars, we are able to manipulate the shape of flow fields (Fig. 4F-G) (Video 9, Video 10, Video 11). We are able to model the large scale structures of the observed flow fields with series of point forces that follow the observed microtubule networks (SI 2.14). Furthermore, we dynamically modulate flow fields by applying a rotating light pattern, creating an "active stir bar" (Fig. 4H) (Video 12). The rotation speed is chosen so that the edge of the light pattern is below the escape velocity found earlier.

In this work, we uncover a set of rules regarding creation and manipulation of active matter structures and resultant fluid flows. Underlying these observations is our capability to isolate active matter into compartments of protein activity using optical boundaries. Utilizing this control, we are able to measure properties of active matter that were previously unattainable, such as scaling of size and speed of networks, and the generated flow fields. Our system provides precise creation, movement, and merging of non-equilibrium structures, analogous to the exquisite control demonstrated by biological systems. Understanding and mimicking this control has been a long-standing open area of research as it offers the promise to recreate complex biological phenomena such as movement, division, and cytoplasmic streaming. Our work takes a step towards this goal by creating dynamic compartments of active structures with light to gain control over their non-equilibrium 

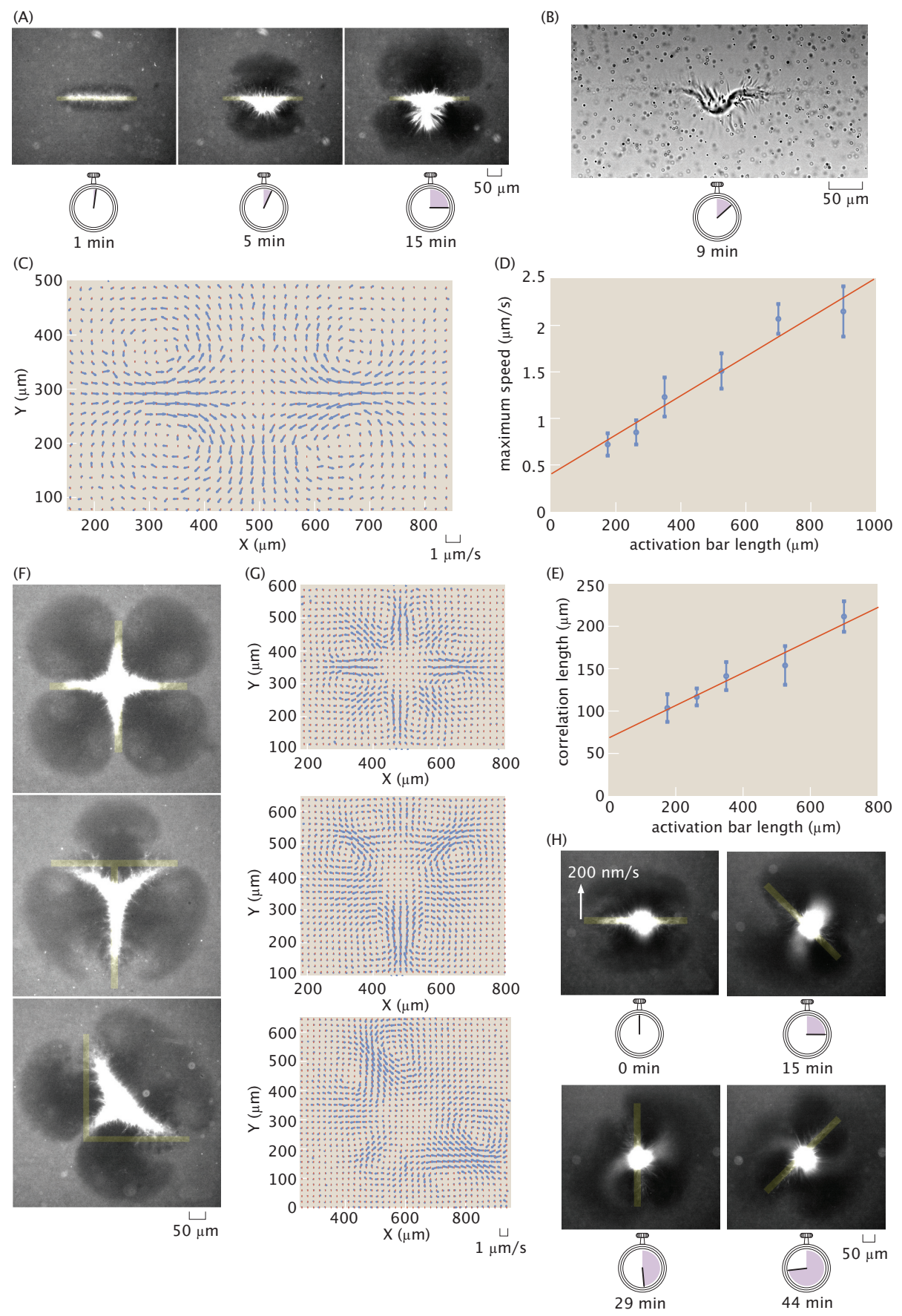

Figure 4: Advective fluid flow is created and controlled with patterned light. (A) Microtubule organization created by a $350 \mu \mathrm{m}$ x $20 \mu \mathrm{m}$ light pattern. Time series demonstrate continuous contraction of microtubules towards the pattern center along the major axis. (B) Brightfield image of A shows a contracting microtubule network and tracer particles used to visualize fluid flow. (C) Time-averaged flow field of A. (D) Averaged maximum flow speed as a function of activation bar length. (E) Averaged correlation length (size) of flow field as a function of activation bar length. (F) Superposition of excitation bars generate different patterns of contractile microtubules. (G) Corresponding time-averaged fluid flow fields. (H) Time lapse of a light pattern rotating with an edge speed of $200 \mathrm{~nm} / \mathrm{s}$. In C, D, E, and G, flow fields are of the background buffer as measureed by particle tracking velocimetry. In $\mathrm{D}$ and $\mathrm{E}$, the average is over 9 experiments and the error bars are the associated standard deviations. The red line is a linear fit to the data. 
properties. Future work will measure what sets the size and speed limits of non-equilibrium structures and the interplay of mass flows and structural changes. Such results may be tested against existing theoretical work on cytoskeletal mechanics and used to develop new theories. Broader exploration of this system's capabilities and limits will yield additional insight into active matter phenomenology and how it may be sculpted to accomplish tasks. 
Acknowledgements The authors would like to thank Maya Anjur-Dietrich, John Brady, Jehoshua Bruck, Vahe Galstyan, Soichi Hirokawa, Christina Hueschen, Wendell Lim, Wallace Marshall, Dyche Mullins, Dan Needleman, Paul Rothemund, and Erik Winfree for influential scientific discussions. We thank Lukasz Bugaj, Zvonimir Dogic, Adam Frost, Walter Huynh, Rustem Ismagilov, Linnea Metcalf, Henry Nguyen, and Ron Vale for advice and assistance during development of the experimental system. Paul Sternberg for use of a microscopy system for initial light activation experiments. We are grateful to Nigel Orme for assistance with figures and illustrations. The authors would like to acknowledge support from the NIH through grants 1R35 GM118043-01 (RP) and NIH DP5 OD012194 (MT); the NSF through NSF 1330864 (MT); the John Templeton Foundation as part of the Boundaries of Life Initiative Grants 51250 \& 60973 (RP); The Foundational Questions Institute and Fetzer Franklin Fund through FQXi 1816 (RP, MT); and the UCSF Center for Systems and Synthetic Biology NIGMS P50 GM081879 (MT).

\section{Methods and Materials}

\subsection{Kinesin Chimera Construction and Purification}

To introduce optical control, we implemented the light-induced hetero-dimer system of iLid and SspB-micro [27]. We constructed two chimeras of drosophila kinesin K401: K401-iLid and K401micro (Fig S1).

\section{K401-iLID

Kinesin Heavy Chain (401) iLID His

K401-micro

His-MBP $>$ Kinesin Heavy Chain (401) micro

Figure S1: Kinesin motor coding regions

To construct the K401-iLID plasmid, we PCR amplified the coding region of iLID from the plasmid pQE-80L iLID (gift from Brian Kuhlman, Addgene 60408) and used Gibson assembly to insert it after the C-terminus of K401 in the plasmid pBD-0016 (gift from Jeff Gelles, Addgene 15960). To construct the K401-micro plasmid, we PCR amplified the coding region of K401 from the plasmid pBD-0016 and used Gibson assembly to insert it in between the His-MBP and micro coding regions of plasmid pQE-80L MBP-SspB Micro (gift from Brian Kuhlman, Addgene 60410). As reported in [27], the MBP domain is needed to ensure the micro domain remains fully functional during expression. Subsequent to expression, the MBP domain can be cleaved off by utilizing a TEV protease site.

For protein purification, we used the His tags that were provided by the base plasmids. For protein expression, we transformed the plasmids into BL21(DE3)pLysS cells. The cells were induced at OD 0.6 with $1 \mathrm{mM}$ IPTG and grown for 16 hours at $18^{\circ} \mathrm{C}$. The cells were pelleted and then resuspended in lysis buffer ( $50 \mathrm{mM}$ sodium phosphate, $4 \mathrm{mM} \mathrm{MgCl} 2,250 \mathrm{mM} \mathrm{NaCl}, 25 \mathrm{mM}$ imidazole, 0.05 
mM MgATP, $5 \mathrm{mM} \mathrm{BME,} 1 \mathrm{mg} / \mathrm{ml}$ lysozyme and 1 tablet/50 mL of Complete Protease Inhibitor). After an hour, the lysate was passed through a $30 \mathrm{kPSI}$ cell disruptor to lyse any remaining cells. The lysate was then clarified by an ultra-centrifuge spin at 30,000 g for 1 hour. The clarified lysate was incubated with Ni-NTA agarose resin (Qiagen) for 1 hour. The lysate mixture was loaded into a chromatography column, washed three times with wash buffer (lysis buffer without the lysozyme and protease inhibitor), and eluted with $500 \mathrm{mM}$ imidazole. For the K401-micro elution, we added TEV protease at a 1:25 mass ratio to remove the MBP domain. Protein elutions were dialyzed overnight using a $30 \mathrm{kDa}$ MWCO membrane to reduce trace imidazole and small protein fragments. Protein was concentrated with a centrifugal filter (Amicon) to $8-10 \mathrm{mg} / \mathrm{ml}$, as determined by absorption of $280 \mathrm{~nm}$ light with a UV spectrometer.

\subsection{Microtubule Polymerization and Length Distribution}

We polymerized tubulin with the non-hydrolyzable GTP analog GMP-CPP, using a protocol based on the one found on the Mitchison lab homepage [28]. A polymerization mixture consisting of M2B buffer (80 mM K-PIPES pH 6.8, 1 mM EGTA, $2 \mathrm{mM} \mathrm{MgCl2),} 75 \mu \mathrm{M}$ unlabeled tubulin (PurSolutions), $5 \mu \mathrm{M}$ tubulin-AlexaFluor647 (PurSolutions), $1 \mathrm{mM}$ DTT, and 0.6 mM GMP-CPP (Jenna Biosciences) was spun at $\sim 300,000 \mathrm{~g}$ for 5 minutes at $2^{\circ} \mathrm{C}$ to pellet aggregates. The supernatant was then incubated at $37^{\circ} \mathrm{C}$ for 1 hour to form GMP-CPP stabilized microtubules.

To measure the length distribution of microtubules, we imaged fluorescently labeled microtubules immobilized onto the cover glass surface of a flow cell. The cover glass was treated with a $0.02 \%$ solution of poly-L-lysine (Sigma) to promote microtubule binding. The lengths of microtubules were determined by image segmentation. To reduce the effect of the non-uniformity in the illumination, we apply a Bradley adaptive threshold with a sensitivity of 0.001 and binarize the image. Binary objects touching the image border and smaller than 10 pixels in size were removed. To connect together any masks that were "broken" by the thresholding, a morphological closing operation was performed with a 3 pixel $\times 3$ pixel neighborhood. Masks of microtubules are then converted into single pixel lines by applying a morphological thinning followed by a removal of pixel spurs. The length of a microtubule is determined by counting the number of pixels that make up each line. The histogram of lengths was fit to an exponential distribution to determine the mean length (Fig. S2). We note that stabilized microtubules typically follow a log-normal distribution, however, we believe that our truncation of the lower microtubule length bins leads to an exponential distribution instead. 


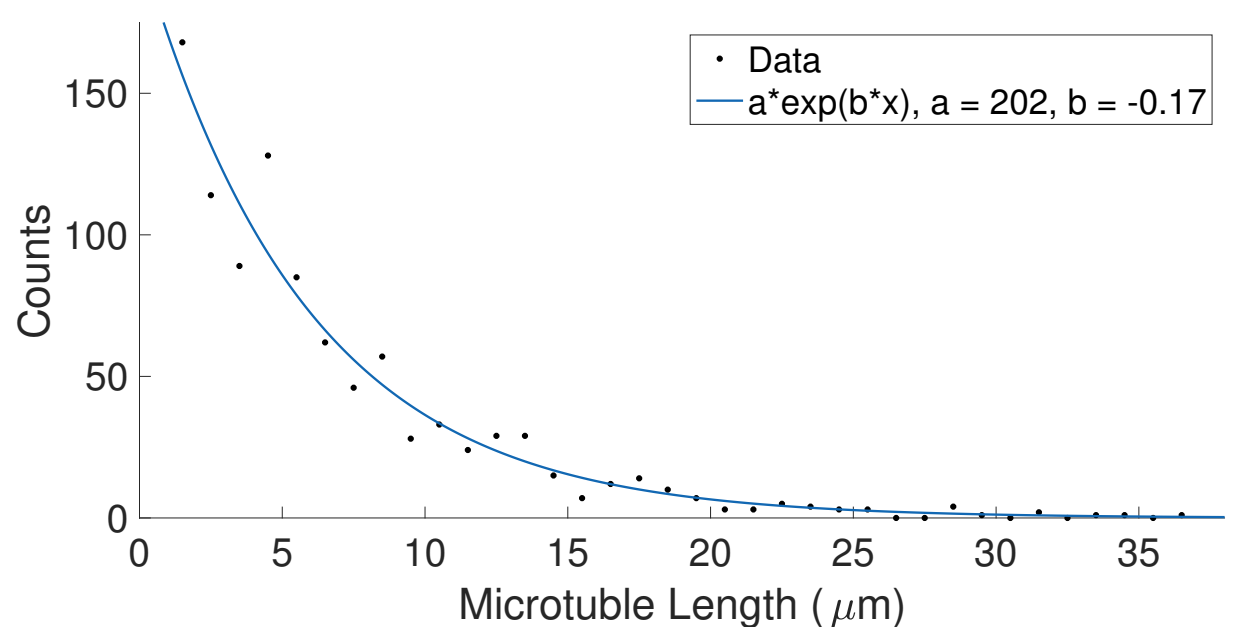

Figure S2: Length distribution of microtubules. Fitting of data to an exponential distribution gives a mean length of $\sim 6 \mu \mathrm{m}$.

\subsection{Flow Cell Preparation and Construction}

Microscope slides and cover glass are passivated against non-specific protein absorption with a hydrophilic acrylamide coating [29]. The glass is first cleaned in a multi-step alkaline etching procedure that removes organics and the surface layer of the glass. The slides and cover glass are immersed and sonicated for 30 minutes successively in 1\% Hellmanex III (Helma Analytics) solution, followed by ethanol, and finished in $0.1 \mathrm{M} \mathrm{KOH}$ solution. After cleaning, the glass is immersed in a silanizing solution of $98.5 \%$ ethanol, $1 \%$ acetic acid, and $0.5 \% 3$-(Trimethoxysilyl)poropylmethacrylate (Sigma) for 10-15 min. After rinsing, the slides are immersed overnight in a degassed $2 \%$ acrlylamide solution with $0.035 \%$ TEMED and $3 \mathrm{mM}$ ammonium persulfate. Just before use, the glass is rinsed in distilled water and nitrogen dried. Parafilm gaskets with pre-cut $3 \mathrm{~mm}$ wide channels are used to seal the cover glass and slide together, making a flow cell. After the addition of the reaction mixture, a flow cell lane is sealed with a fast setting silicone polymer (Picodent Twinsil Speed).

\subsection{Reaction Mixture and Sample Preparation}

K401-micro , K401-iLid, and microtubules were combined into a reaction mixture, leading to final concentrations of $1 \mu \mathrm{M}$ of each motor and 1.5-2.5 $\mu \mathrm{M}$ of tubulin. To minimize unintended light activation, the sample was prepared under dark-room conditions, where the room light was filtered to block wavelengths below $580 \mathrm{~nm}$ (Kodak Wratten Filter No. 25). The base reaction mixture provided a buffer, an energy source (MgATP), a crowding agent (glycerol), a surface passivating polymer (pluronic F127), oxygen scavenging components to reduce photobleaching (glucose oxidase, glucose, catalase, trolox, DTT), and ATP-recycling reagents to prolong motor activity (pyruvate kinase/lactic dehydrogenase, phosphoenolpyruvic acid). The reaction mixture consisted of $58.4 \mathrm{mM}$ K-PIPES pH 6.8, $2.6 \mathrm{mM}$ potassium phosphate, $3.2 \mathrm{mM}$ potassium chloride, $0.73 \mathrm{mM}$ EGTA, 4.7 mM MgCl2, $1.4 \mathrm{mM} \mathrm{MgATP} \mathrm{(Sigma),} 10 \%$ glycerol, $0.50 \mathrm{mg} / \mathrm{mL}$ pluronic F127 (Sigma), 0.22 $\mathrm{mg} / \mathrm{ml}$ glucose oxidase (Sigma), $3.2 \mathrm{mg} / \mathrm{ml}$ glucose, $0.038 \mathrm{mg} / \mathrm{ml}$ catalase (Sigma), $5.4 \mathrm{mM}$ DTT, 
bioRxiv preprint first posted online Dec. 21, 2018; doi: http://dx.doi.org/10.1101/504456. The copyright holder for this preprint

(which was not peer-reviewed) is the author/funder, who has granted bioRxiv a license to display the preprint in perpetuity.

All rights reserved. No reuse allowed without permission.

$2.0 \mathrm{mM}$ trolox (Sigma), 0.026 units pyruvate kinase/lactic dehydrogenase (Sigma P0294), and 26.6 mM phosphoenolpyruvic acid (Beantown Chemical 129745).

\subsection{Gliding Assay Sample Preparation}

Alkaline cleaned cover glass and ethanol scrubbed slides were prepared and $5 \mu \mathrm{L}$ flow chambers were prepared with doubled sided tape. Motors were bound to the surface by successive incubations of the chamber with $400 \mu \mathrm{g} / \mathrm{mL}$ penta-His antibody (Qiagen) for $5 \mathrm{~min}, 10 \mathrm{mg} / \mathrm{ml}$ whole casein (Sigma) for $5 \mathrm{~min}$, and finally motor protein $(1 \mathrm{mg} / \mathrm{mL}$ in M2B) for $5 \mathrm{~min}$. Unbound motors were washed out with M2B buffer, then AlexaFluor 647 labeled GMP-CPP stabilized microtubules in M2B with $5 \mathrm{mM} \mathrm{MgATP}$ and $1 \mathrm{mM}$ DTT were flowed in.

\subsection{Preparation of Tracer Particles}

To measure the fluid velocity, we used $1 \mu \mathrm{m}$ polystyrene beads (Polysciences) as tracer particles. To passivate the hydrophobic surface of the beads, we incubated them overnight in M2B buffer with 50 $\mathrm{mg} / \mathrm{ml}$ of F127 pluronic. Just before an experiment, the pluronic coated beads are washed by pelleting and resuspending in M2B buffer with $0.5 \mathrm{mg} / \mathrm{ml}$ pluronic to match the pluronic concentration of the reaction mixture.

\subsection{Microscope Instrumentation}

We performed the experiments with an automated widefield epifluorescence microscope (Nikon TE2000). We custom modified the scope to provide two additional modes of imaging: epi-illuminated pattern projection and LED gated transmitted light. We imaged light patterns from a programmable DLP chip (EKB TEchnologies DLPß LightCrafter ${ }^{\top M}$ E4500 MKII ${ }^{\top M}$ Fiber Couple) onto the sample through a user-modified epi-illumination attachment (Nikon T-FL). The epi-illumination attachment had two light-path entry ports, for light paths allowed for two different light paths on the which combined the projected pattern light path with the standard widefield epi-fluorescence light path by a dichroic mirror (Semrock BLP01-488R-25). The magnification of the epi-illuminating system was designed so that when the entire DLP chip was on, the imaging sensor of the camera (FliR BFLY-U3-23S6M-C) was fully illuminated. Experiments were run with Micro-Manager [30], running custom scripts to controlled pattern projection and stage movement. For the transmitted light path, we replaced the standard white-light brightfield source (Nikon T-DH) with an electronically time-gated $660 \mathrm{~nm}$ LED (ThorLabs M660L4-C5). This was done to minimize light-induced dimerization during bright field imaging.

\section{Data Acquisition and Analysis}

\subsection{Microscopy Protocol}

Samples were imaged at 10X (Fig 1C, 1E, 1F, 2D, 4A, 4F, and 4H) or 20X (Fig. 1D, 2B, 3B, 3D, and $3 \mathrm{E})$. For Figures $2 \mathrm{E}$ and $2 \mathrm{~F}$, the distance span of the merger experiments required us to pool data 
taken at 10X (500 $\mu \mathrm{m}$ and $1000 \mu \mathrm{m}$ separations) and 20X magnifications $(175 \mu \mathrm{m}, 250 \mu \mathrm{m}$, and 350 $\mu \mathrm{m}$ separations). For the formation, merging, and movement experiments represented in Figures $1-3$, the images of the fluorescent microtubules were acquired every $20 \mathrm{~s}$. For the flow experiments represented in Figure 4, a brightfield image and subsequent fluorescent image were acquired every 4 seconds to observe the tracer particles and microtubules, respectively. The increased frame rate was needed to ensure sufficient accuracy of the particle velocimetry. For all experiments, we activated light-induced dimerization in the sample every $20 \mathrm{~s}$ with a brief $300 \mathrm{msec}$ flash of $2.4 \mathrm{~mW} / \mathrm{cm}^{2}$ activation light from a $\sim 470 \mathrm{~nm}$ LED. The rate of activation was based on the estimated offrate of the iLid-micro complex [27] of $\sim 30 \mathrm{~s}$. The duration of the activation light was empirically determined, by gradually increasing the time in 50 msec increments until we observed the formation of an aster. Samples were imaged for up to 1 hour. Typically, one experiment was run per flow cell lane. We placed these time limitations on the sample viewing to minimize effects related to cumulative photobleaching, ATP depletion, and unintended activity of the light-dimerizable proteins. After several hours, unactivated "dark" regions of the sample begin to show bundling of microtubules.

\subsection{Determining Aster Size}

We find the size of the aster through image segmentation and then use the perimeter of the segmented region to determine the diameter of the aster. Before segmenting the aster, we first identify the region of activation. We define the region of activation as the disk encompassing the aster and the region devoid of microtubules around the aster. To identify this region, we segment this low intensity region around the aster. The low intensity region around the aster is found by subtracting the final frame of aster formation from the first frame of the image acquisition. After subtraction, the void region is the brightest component of the image. We segment this region by performing an intensity and size threshold to create a mask. The aster-shaped hole in the mask is then filled. Using the perimeter of the mask, we calculate the diameter of the disk region of activation.

Asters are segmented through an intensity-based method. We begin by subtracting the initial frame of the acquisition from the final frame of aster formation. After the image subtraction, the brightest component of the image is the aster. The resulting image is then binarized with a highpass intensity threshold. After the threshold is applied there are multiple binary objects due to fluorescent aggregates. To segment the aster from the binary mask, we isolate the disk region of activation, and perform an object size threshold. We find the diameter of the aster by calculating the distance between the two furthest points on the perimeter of the aster binary mask.

\subsection{Diffusion Coefficient of a Microtubule}

We estimate the diffusion coefficient for a single microtubule to compare with the effective diffusion coefficient we estimate for aster decay. The diffusion coefficient $D$ for an object in liquid media can be calculated from the drag coefficient $\gamma$

$$
D=\frac{k_{B} T}{\gamma},
$$

where $k_{B}$ is the Boltzmann constant and $\mathrm{T}$ is the temperature, for which we use $298 \mathrm{~K}$. We model a microtubule as a $6 \mu \mathrm{m}$, the average microtubule length that we measure (SI. 1.2), long cylinder 
with a radius of $12.5 \mathrm{~nm}$. The drag coefficients for a cylinder have been found previously [31] for motion either parallel $\gamma_{\|}$or perpendicular $\gamma_{\perp}$ to the long axis of the cylinder

$$
\begin{aligned}
\gamma_{\|} & =\frac{2 \pi \eta L}{\ln (L / 2 r)-0.20}, \\
\gamma_{\perp} & =\frac{4 \pi \eta L}{\ln (L / 2 r)+0.84} .
\end{aligned}
$$

Here, $L$ is the length of the cylinder, $r$ is its radius, and $\eta$ is the viscosity of the fluid, which we estimate to be $1.85 \times 10^{-3} \mathrm{~Pa} \cdot \mathrm{s}$ (SI 2.12). Using these equations, we calculate diffusion coefficients for parallel $D_{\|}$and perpendicular $D_{\perp}$ motion. Using the parameters detailed above, we calculate $D_{\|}=$ $0.311 \mu \mathrm{m}^{2} / \mathrm{s}$ and $D_{\perp}=0.186 \mu \mathrm{m}^{2} / \mathrm{s}$. We assume that the larger diffusion coefficient dominates and thus use $D_{\|}$, the longitudinal diffusion coefficient, as the diffusion coefficient for a single microtubule in Fig. 1E.

\subsection{Scaling Arguments for Aster Size and Comparison to Data}

We consider how the total number of microtubules in an aster relates to the volume of the projected light pattern. We are projecting a disk pattern of light on the sample from below. The channel is a constant height, $z \approx 70 \mu \mathrm{m}$. We therefore treat the light excitation volume as a cylinder $V_{\text {light }}=\frac{1}{4} \pi z d_{\text {light }}^{2}$ where $d_{\text {light }}$ is the diameter of the excitation disk. If we look at experimental data, we see evidence of a linear relationship between the light volume and the number of microtubules that are present during aster formation (Fig. S3A). The implication of this observation is that the density $\rho$ of microtubules is uniform. Furthermore, we see that after the initial contraction event, the total integrated fluorescence of the excited region remains constant (Fig. S3B), indicating that the total number of microtubules $N$ is constant during aster formation.

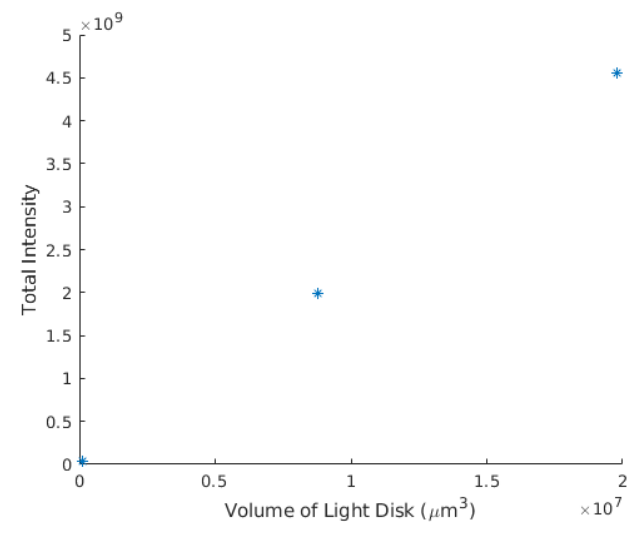

(A)

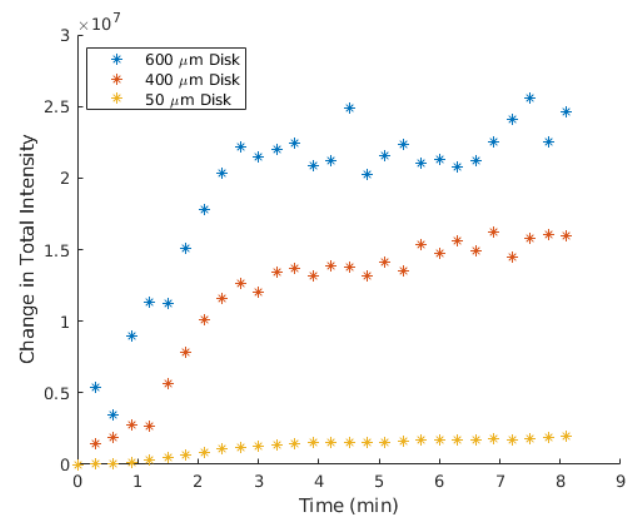

(B)

Figure S3: Measuring the conservation of labeled fluorescent microtubules in the excitation region during aster formation. (A) Total intensity of excitation region as a function of volume of light disk averaged during aster formation. Measurements are for light disks with diameters 50, 400, and 600 $\mu \mathrm{m}$. (B) Change in total intensity inside of the excitation region as a function of time 
Based on these observations we assume the number of microtubules $N$ in the aster is given by

$$
N \approx \rho V_{\text {light }}
$$

We observe that the asters we form are roughly spherical 3D objects, as determined from $\mathrm{z}$ stack imaging. Consequently, we assume the size of the aster $L_{\text {aster }}$ is given by the diameter of the sphere, which scales with microtubule number as

$$
L_{\text {aster }} \propto N^{1 / 3}
$$

and thus

$$
L_{\text {aster }} \propto V_{\text {light }}^{1 / 3}
$$

As noted above, the volume defined by the activation light is a cylinder, then

$$
V_{\text {light }} \propto d_{\text {disk }}^{2}
$$

By combining equations 3 and 4, we arrive at the scaling relationship between aster size and excitation disk size

$$
L_{\text {aster }} \propto d_{\text {disk }}^{2 / 3}
$$

We made a power law fit with a fixed exponent of $2 / 3$ to the data shown in Fig. 1 C. Though we cannot strictly rule out other exponents, we show the fit to demonstrate that the scaling argument determined exponent is at least consistent with the data.

\subsection{Measuring Aster Spatial Distribution with Image Standard Devia- tion}

Image standard deviation is a measure of the spatial width of an intensity distribution over a region of interest, ROI. We use the standard deviation to characterize how the the spatial distribution of microtubules evolves in time. For each time point, we, first, normalize each pixel value, $p(x, y)$ by the total pixel intensity summed across ROI.

$$
p_{\text {norm }}(x, y)=\frac{p(x, y)}{\sum_{x, y \in \mathrm{ROI}} p(x, y)}
$$

where $p(x, y)$ is the raw intensity of the pixel at position $(x, y)$ after background subtraction. We calculate the variance, $\sigma^{2}$, of the intensity distribution as

$$
\sigma^{2}=\sum_{x, y \in \text { ROI }}\left[(x-\bar{x})^{2}+(y-\bar{y})^{2}\right] p_{\text {norm }}(x, y)
$$


where coordinates $\bar{x}$ and $\bar{y}$ are the center of the intensity distribution

$$
\overline{\mathbf{x}}=\sum_{\mathbf{x} \in \mathrm{ROI}} \mathbf{x} p(\mathbf{x})
$$

\subsection{Quantifying Aster Formation and Decay}

\subsubsection{Processing Images for Analysis}

Due to the 3D structure of the aster, we sum over the z-stack for each time point. Next, we process them to correct for the non-uniformity in the illumination and background intensity contribution. We "flatten" the non-uniformity of the image with an image intensity profile found in the following process. We take the first frame of the experiment and perform a morphological opening operation with an 80 pixel disk followed by a Gaussian smoothing with a 20-pixel standard deviation. The resulting image is then normalized to its maximum pixel intensity to generate the image intensity profile. Images are flattened by dividing them by the intensity profile. We note that this strategy depends on there being a uniform density of microtubules in the first frame.

Once images are flattened, the background is found by taking the last frame of aster formation and calculating the mean intensity of the activated region that is devoid of microtubules. Images are subtracted by this background intensity and thresholded so that any negative values are set to zero.

For analyzing the images for the decay process, we alternatively take a region of interest centered on the aster position (from the last frame of aster formation and found using the intensity weighted center) and proportional to the size of the aster in order to reduce the contribution of microtubules diffusing in from the boundary. This proportionality constant was chosen as the ratio of the ROI diameter to the aster diameter for the aster formed with the $50 \mu \mathrm{m}$ disk, which is 1.63 .

\subsubsection{Analysis of Aster Formation}

We interpret the intensity distribution from the images as a measure of the spatial distribution of microtubules. Images are first processed as described in SI. 2.6.1. For aster formation, we take the region of activation as our region of interest as described in (SI. 2.2). We quantify the width of the spatial distribution of the microtubules within the activation region using the image standard deviation, $\sigma(t)$, as a function of time, as described in (SI. 2.5). From our data, we observe $\sigma(t)$ decrease with time (Fig. S4), indicating contraction of the distribution of microtubules. We fit a line to the linear region of $\sigma(t)$. The slope of the line is the reported contraction rate. 


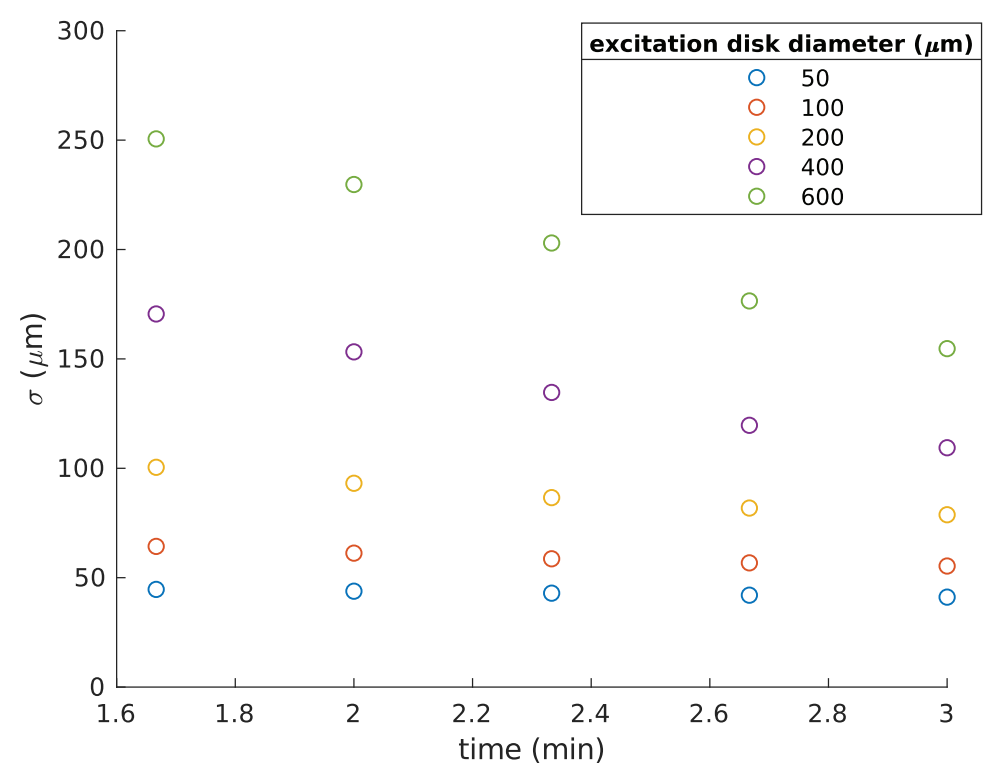

Figure S4: Plot of mean standard deviation of image intensity as a function of time for various excitation disk diameters. Time window is selected for the linear regime.

\subsubsection{Analysis of Aster Decay}

When the activation light is removed, the iLid-micro dimer begins to disassociate, leading to uncrosslinked microtubules. From the last three image snap shots of Fig. 1C, we see the arms of the asters begin to dissolve into unresolved clouds of microtubules. We expect that these microtubules are free to move in solution.

For an ideal 2D Gaussian distribution of diffusing particles starting with a finite radius of $w$, we expect

$$
p(r, t)=\frac{1}{\pi\left(4 D t+w^{2}\right)} e^{-r^{2} /\left(4 D t+w^{2}\right)},
$$

where $D$ is the diffusion constant.

The variance $\sigma_{\text {Gauss }}^{2}$ of this distribution as a function of time $t$ is given by

$$
\sigma_{\text {Gauss }}^{2}(t)=4 D t+w^{2}
$$

The variance $\sigma_{\text {Gauss }}^{2}$ increases linearly with $t$ with a slope of $4 D$.

We characterize the aster decay process by measuring the variance of the experimentally observed intensity distributions as a function of time. Images are first processed as described in SI. 2.6.1, then the spatial distribution is found (SI.2.5). Although our spatial distributions are not strictly Gaussian, we observe that for our data the variance $\sigma^{2}$, as defined in SI 2.5, increase linearly with $t$ (Fig. S5), which suggests that the decay process is described by the diffusion of unbound microtubules. By analogy to the 2D ideal Gaussian case, we calculate an equivalent diffusion coefficient of our distributions by a linear fit of the variance versus time and finding $D$ from the slope. This gives us a diffusion coefficient in units of $\mu \mathrm{m}^{2} / \mathrm{s}$. 


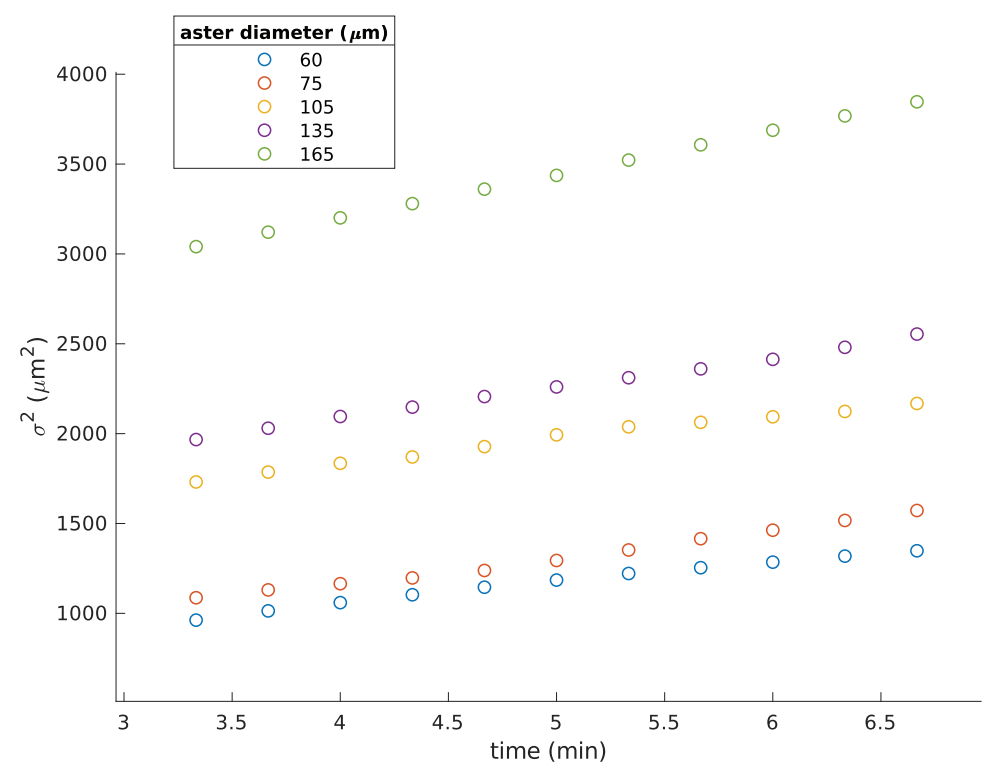

Figure S5: Plot of mean variance of image intensity as a function of time for different initial aster sizes. Time window is selected for the linear regime. The measure of time is relative to the beginning of aster decay.

\subsection{Reversibility of Aster Formation and Decay}

To show that aster decay is driven by motors reverting to monomers as opposed to irreversible events such as ATP depletion or the denaturing of protein, we provide an illustrative experiment of aster formation followed by decay followed again by aster formation. 


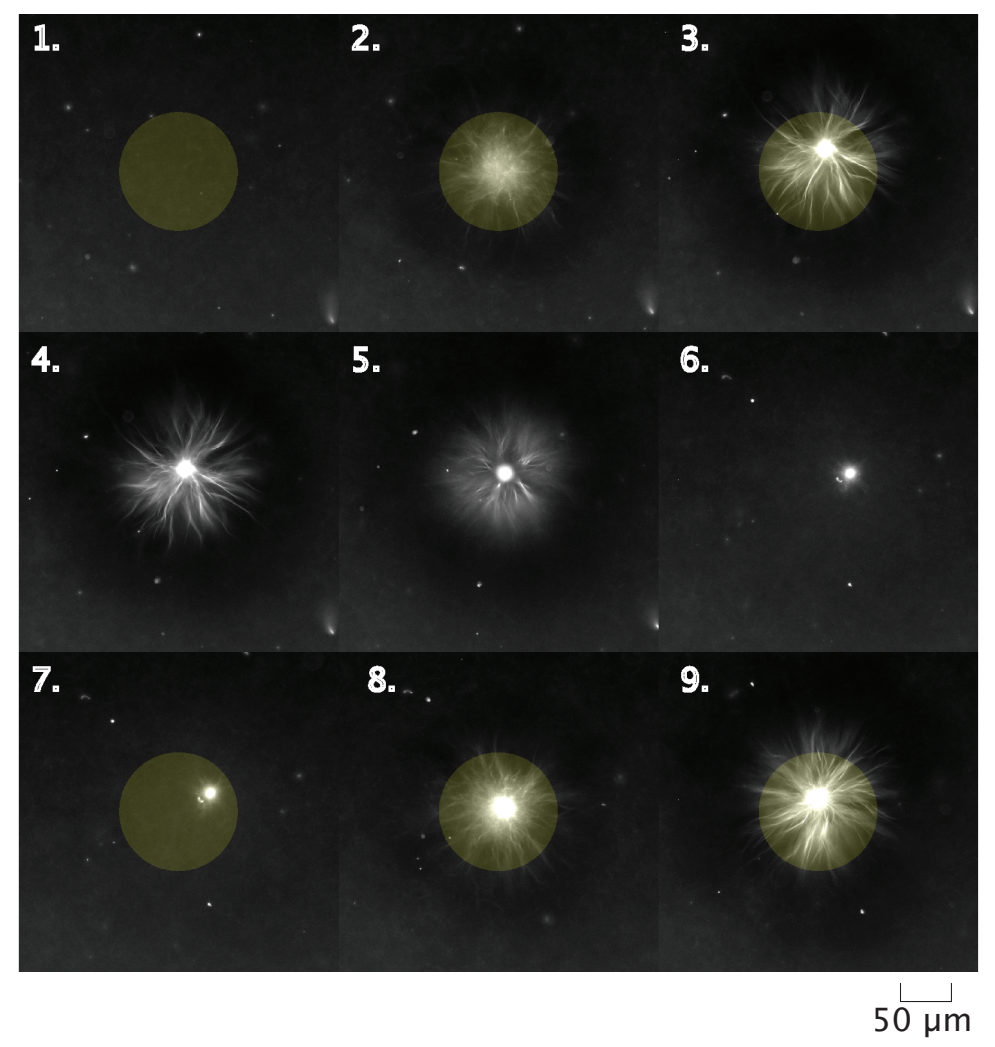

Figure S6: Time series of light induced aster formation, decay, then formation. First formation frames are at time points $\mathrm{t}=(1) 0,(2) 6.7$, and (3) $16.3 \mathrm{~min}$. Aster decay frames are for $\mathrm{t}=(4)$ 16.7, (5) 25, and (6) $112.7 \mathrm{~min}$. Second aster assembly frames are $\mathrm{t}=(7)$ 113, (8) 120, and (9) $129.3 \mathrm{~min}$

\subsection{Single Motor Velocity Determination from Gliding Assay}

Gliding assay images were acquired every second with total internal reflection fluorescence (TIRF) microscopy. Motor speeds were determined by tracking individual microtubules. Single microtubules were identified by edge detection followed by size thresholding to remove small particles on the glass and large objects that are overlaying microtubules. The centroid of each object is identified and paired with the nearest-neighbor in the next frame. The Euclidian distance between the paired centroids is calculated and used to determine the microtubule velocity. The distribution of frame-by-frame velocities (excluding those less than $75 \mathrm{~nm} / \mathrm{s}$, which is our typical sample drift) was fit to an exponential distribution to obtain the mean velocity. 
(A)

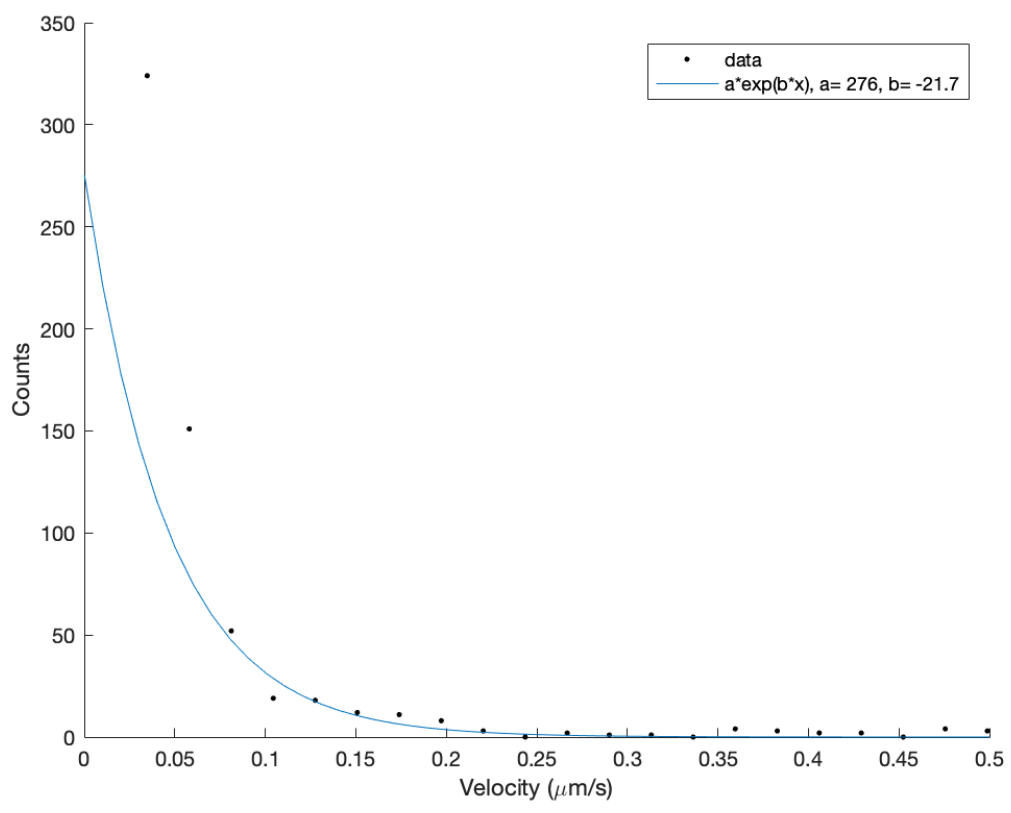

(B)

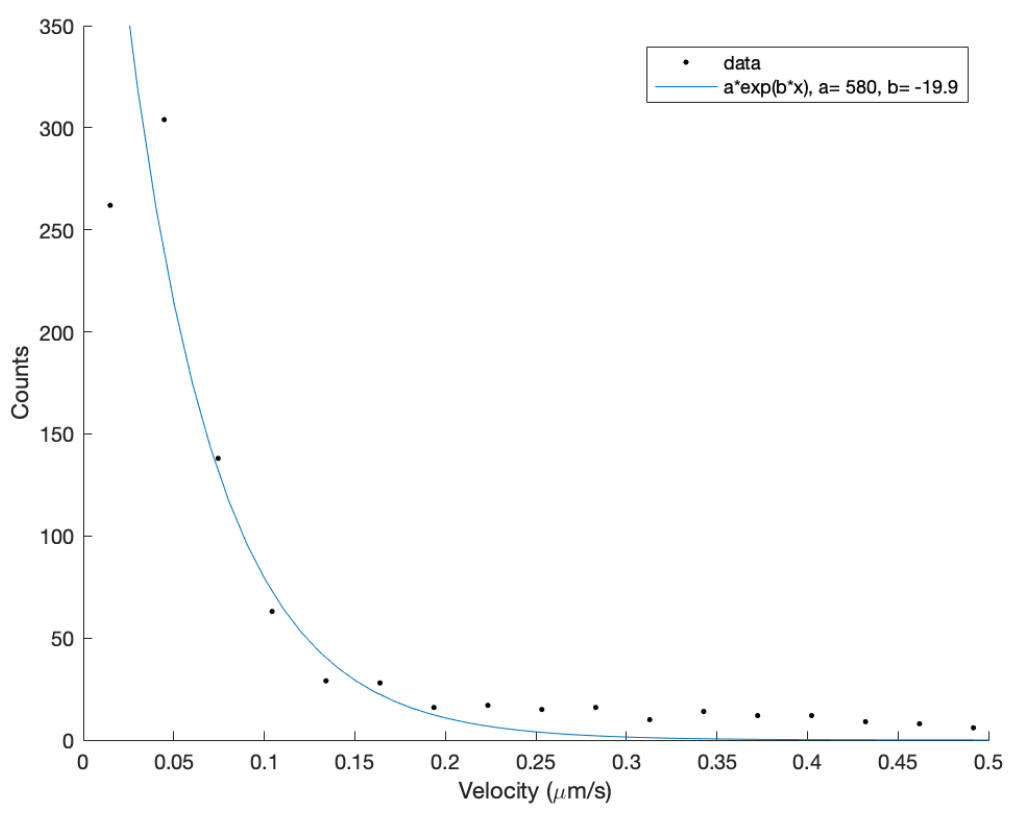

Figure S7: Velocity distribution of gliding microtubules. (A) Distribution for K401-iLid motors. Fitting of data to an exponential distribution $\left(\mathrm{R}^{2}=0.913\right)$ gives a mean velocity of $210 \pm 30 \mathrm{~nm} / \mathrm{s}$. (B) Distribution for K401-micro motors. Fitting of data to an exponential distribution $\left(\mathrm{R}^{2}=0.917\right)$ gives a mean velocity of $270 \pm 30 \mathrm{~nm} / \mathrm{s}$. 


\subsection{Effective Potential of a Moving Aster}

We observe when the light pattern stops moving at speed $v_{\text {light }}$, the aster immediately returns to the center of the light pattern at speed $v_{\text {return }}$. From the data (Fig. S8), we see that

$$
v_{\text {return }} \approx v_{\text {light }}
$$

This is the behavior expected for an object under the influence of a potential at low-Reynoldsnumber, where the aster has negligible momentum and the forces are essentially instantaneous. This observation supports the notion that a moving aster can be modeled as being in an effective potential.

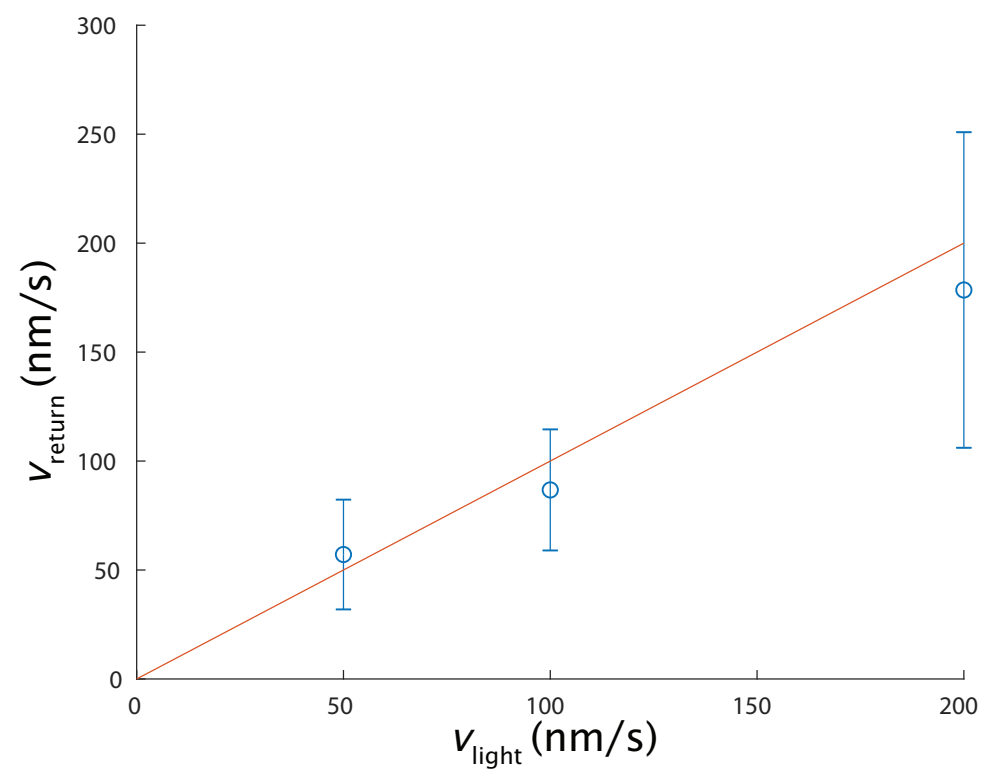

Figure S8: The speed at which an aster returns to the center of an excitation disk once the disk stops moving. Red line is a plot of $\mathrm{y}=\mathrm{x}$.

We estimate the potential and the forces acting on a moving aster from the viscous drag of the background fluid, in an analogous way to how this done for objects trapped in an optical tweezer [32]. If we assume the aster is a spherical object of radius $a$ and is moving with speed $v_{\text {light }}$, it will experience a viscous drag force $F_{\text {drag }}$ :

$$
F_{\text {drag }}=6 \pi \eta a v_{\text {light }},
$$

where $\eta$ is the fluid viscosity. $F_{\text {drag }}$ is equal to the force $F_{\text {pull }}$ that is pulling the aster towards the light pattern. From the results of Fig. $2 \mathrm{C}$, we note the observed distance shift $\ell$ of the aster from the center of the moving light pattern is roughly linear with excitation disk movement speed $v_{\text {light }}$. The linearity of $\ell$ versus $v_{\text {light }}$ implies that $F_{\text {pull }}$ acts like a spring:

$$
F_{\text {pull }} \approx k_{\text {spring }} \ell,
$$

where $k_{\text {spring }}$ is the spring constant. Setting these two forces equal gives a spring constant of

$$
k_{\text {spring }} \approx \frac{6 \pi \eta a v_{\text {light }}}{\ell} .
$$


The effective potential $U_{\text {pull }}$ for this force is

$$
U_{\text {pull }}=\frac{1}{2} k_{\text {spring }} \ell^{2}
$$

The aster in Fig. $2 \mathrm{C}$ is $\approx 25 \mu \mathrm{m}$ in diameter. Assuming that $\eta \approx 2 \times 10^{-3} \mathrm{~Pa} \cdot \mathrm{s}$ (SI 2.12), we find that $k_{\text {spring }} \approx 3 \mathrm{fN} / \mu \mathrm{m}$, roughly 300 times weaker than a typical optical tweezer. For the maximum observed displacement of $\ell \approx 30 \mu \mathrm{m}$, the energy stored in the potential, or equivalently, the work done by the system to return the aster back to the center of the light pattern is $\approx 300 k_{B} T$.

\subsection{Aster Tracking}

For each time point, we sum over the z-stack to form a single image. The image is then passed through a morphological top-hat filter with a structure element of a 100 pixel disk to "flatten" nonuniformities in the illumination. The image is then projected into a $1 \mathrm{D}$ intensity profile. We project onto the $\mathrm{x}$-axis by summing along the line that passes through the center of the excitation disk with a 100 pixel window in y. Aster centers are then found at each frame by fitting the intensity profiles to Gaussian functions.

For 2D tracking, the movement of the aster is found by comparing the centroid of the aster in each frame. The raw images are processed using a Gaussian filter with a standard deviation of 1 pixel, followed by thresholding to eliminate the background noise.

\subsection{Fluid Flow Patterns and Velocity Fields}

The fluid flow generated by the movement of microtubule filaments is measured using Particle Tracking Velocimetry (PTV) [33] of fiducial tracer particles. Inert $1 \mu \mathrm{m}$ diameter microspheres (SI 1.6) are added to the reaction buffer and imaged with brightfield microscopy. The images are pre-processed using a Gaussian filter with a standard deviation of 1 pixel, followed by thresholding to eliminate the background noise. After filtering, the centroid of each particle is measured and tracked.

A nearest-neighbor algorithm [34] is applied to find particle pairs within a square search window (30 pixels). Displacement vectors are then calculated by comparing the position of particle pairs in consecutive frames. The same process is repeated for the entire image sequence (30 min). The velocity field is generated by dividing the displacement vector field by the time interval between frames. The averaged velocity field shown in Fig. 4 is carried out by grouping and averaging all velocity vectors within a 30 pixel $\times 30$ pixel window.

In order to understand how the flow field changes over time, we recalculate the average velocity field, dividing the 30 minute experiment into four 7.5 minute time windows. The resulting velocity fields are shown in (Fig. S9). We note that the structure of the flow field remains similar throughout the experiment. In addition, the maximum speed of the velocity field is constant over time (Fig. S10), which further confirms that the fluid flow is stable over the experiment. 
(A)
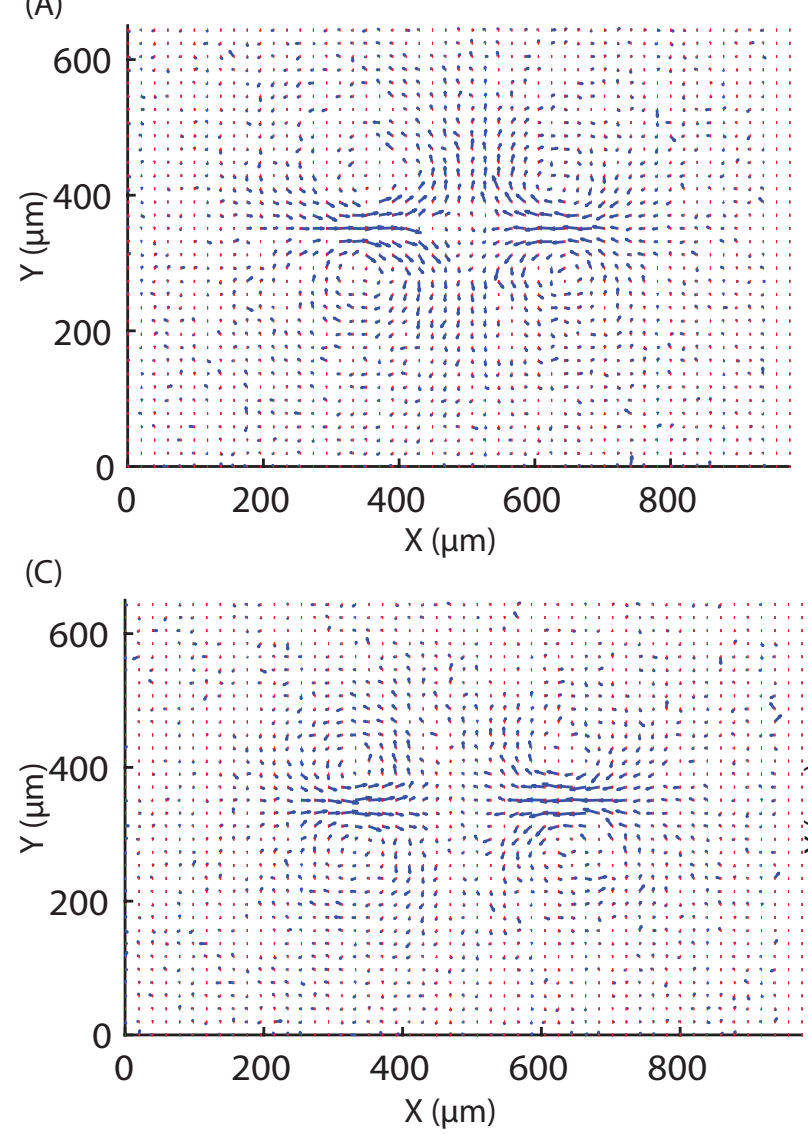
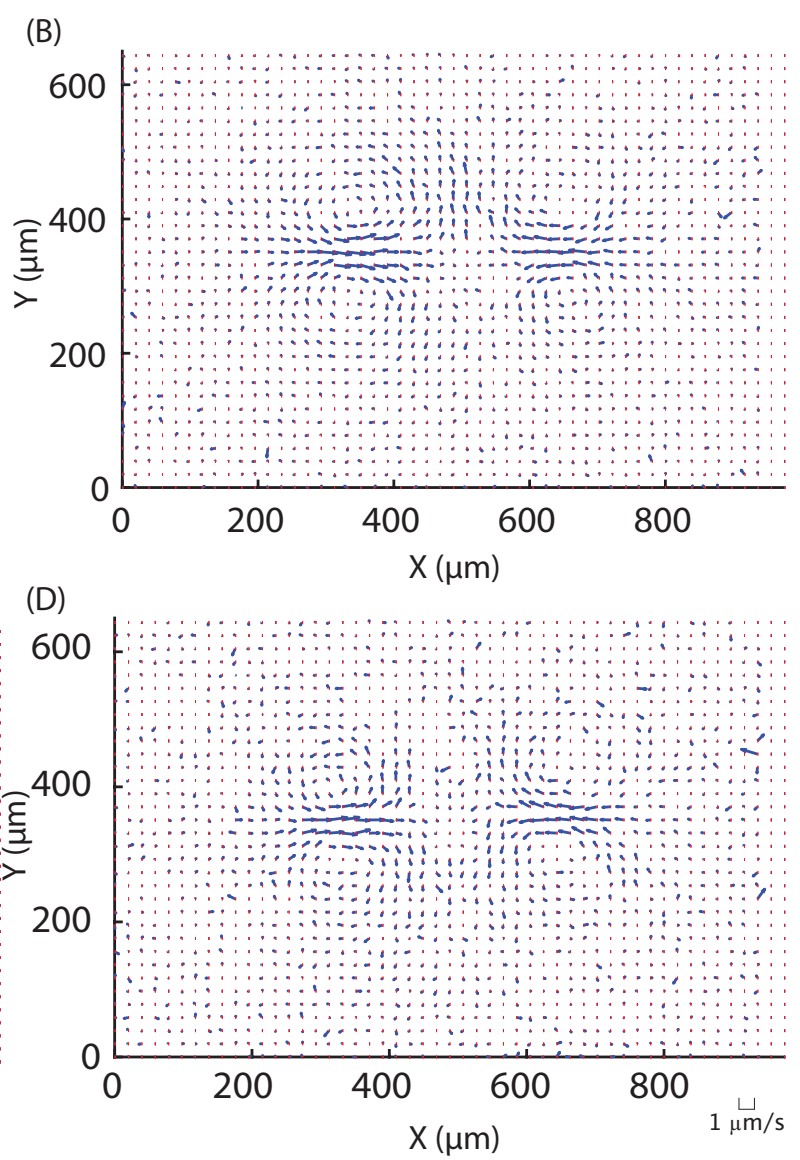

Figure S9: Velocity field averaged over 7.5 minute intervals in a single experiment. Time windows are $(\mathrm{A}) \mathrm{t}=0-7.5 \mathrm{~min},(\mathrm{~B}) \mathrm{t}=7.5-15 \mathrm{~min},(\mathrm{C}) \mathrm{t}=15-22.5 \mathrm{~min}$, and $(\mathrm{D}) \mathrm{t}=22.5-30 \mathrm{~min}$

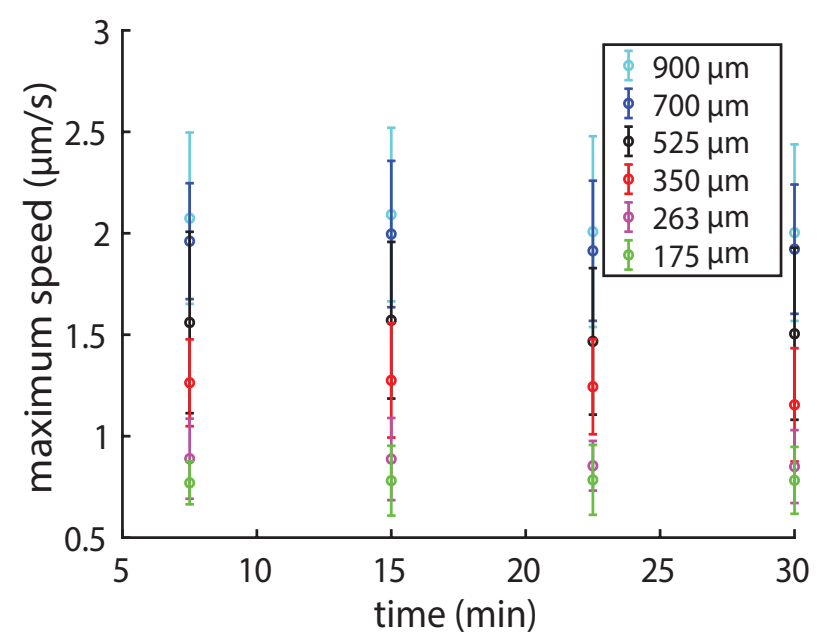

Figure S10: The average maximum speed for four different 7.5 minute time windows. The data points represent the average of nine experiments. The error bars are the associated standard deviation. 


\subsection{Calculating Fluid Viscosity}

To find the viscosity of the background buffer, we used a similar approach to finding the flow fields. We used PTV of fiducial tracer particles (SI 2.11) in inactivated regions of the sample of the 175 $\mu \mathrm{m}$ activation bar experiment. Assuming the buffer is Newtonian [35], the inert tracer particles diffuse freely due to thermal fluctuations. From the tracking results, we measure the mean-squared displacement $\operatorname{MSD}(t)$ of the particles:

$$
\operatorname{MSD}(t)=\left\langle(x(t)-x(0))^{2}+(y(t)-y(0))^{2}\right\rangle,
$$

where $x(t)$ and $y(t)$ are the position of a given particle at time $t$ and \langle\rangle denotes ensemble average. For this calculation, each frame is $t=4 \mathrm{~s}$ apart. The $\operatorname{MSD}(t)$ of a freely diffused particle in 2D follows the Stokes-Einstein equation

$$
\operatorname{MSD}(t)=4 D t=\frac{2 k_{B} T}{3 \pi \eta r} t,
$$

where $r=0.5 \mu \mathrm{m}$ is the radius of the particle. Then, the viscosity of the buffer solution is estimated as

$$
\eta=\frac{8 k_{B} T}{3 \pi r \operatorname{MSD}(t)}
$$

The same process is repeated through nine individual experiments and the average estimated viscosity is $1.85 \times 10^{-3} \mathrm{~Pa} \cdot \mathrm{s}$.

\subsection{Correlation Length}

We use the correlation length as a measure of the spatial size of the flow fields. The distance, $R$, at which velocity vectors become uncorrelated with each other is defined as the correlation length, $L_{c}$. We find the correlation length by first calculating the velocity-velocity correlation coefficient of the averaged velocity field. The correlation coefficient $[6,36]$ is defined as

$$
C(R)=\frac{\langle V(R) \cdot V(0)\rangle}{\left\langle|V(0)|^{2}\right\rangle}
$$

where \langle\rangle denotes assemble average and $\|$ is the magnitude of the vector. By definition, $C(0)=1$. The correlation length $L_{c}$ is defined as the distance when $C\left(L_{c}\right)=0$. The correlation coefficient as a function of $R$ is calculated to determine $L_{c}$ for each bar length (Fig. S11). 


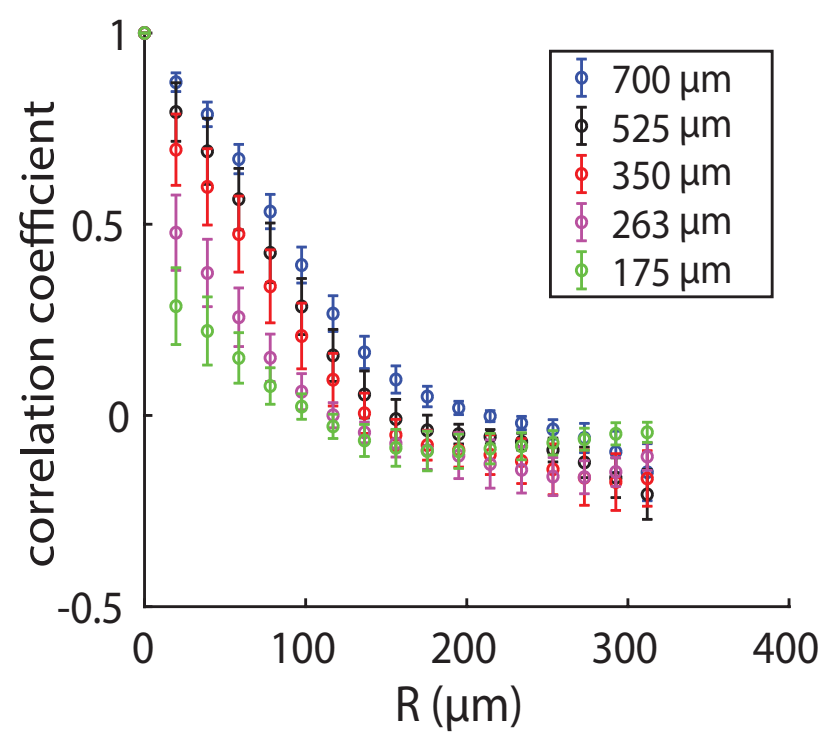

Figure S11: The correlation coefficient as a function of distance. Each marker shows the mean over nine individual experiments and error bars are the associated standard deviation.

\subsection{Theoretical Model of the Fluid Flow Field}

We use solutions of the Stokes equation, the governing equation for fluid flow at low-Reynoldsnumber [37], to model our induced flow fields. One of the simplest solutions of the equation is the Stokeslet, which describes the flow field induced by a point force [38]. Here, we attribute the flowgenerating point forces to contracting microtubule bundles. Since the microtubules at the center of the activation bar appear to contract much more slowly than in other regions of the light pattern, we do not model Stokeslets in the central $120 \mu \mathrm{m}$ of the activation bar. We superimpose the solutions for two series of Stokeslets, one for each side of the bar. Each series of Stokeslets is composed of 7 point forces with identical magnitude $(|\boldsymbol{f}|=0.23 \mathrm{nN})$, separated by $20 \mu \mathrm{m}$ (Fig. S12) to model the $350 \mu \mathrm{m}$ activation bar case.

The velocity field $\boldsymbol{u}(\boldsymbol{x})$ generated by a point force $\boldsymbol{f}$ located at $\boldsymbol{x}^{\prime}$ in a $2 \mathrm{D}$ plane is given as

$$
\boldsymbol{u}(\boldsymbol{x})=\frac{1}{4 \pi \eta}\left(-\boldsymbol{f} \log (r)+\frac{\left(\boldsymbol{f} \cdot\left(\boldsymbol{x}-\boldsymbol{x}^{\prime}\right)\right)\left(\boldsymbol{x}-\boldsymbol{x}^{\prime}\right)}{r^{2}}\right)
$$

where $r$ is the absolute distance, defined as

$$
r=\left|\boldsymbol{x}-\boldsymbol{x}^{\prime}\right|
$$

We note that our model predicts the inflows and outflows for the rectangular bar pattern observed experimentally. However, the vortices are not recovered. We believe this is partially due to the non-Newtonian behavior of the fluid, so applying the Stokes equation will need to be amended for a more complete description. In addition, the mechanism of microtubule induced fluid flow is likely more complex than a simple point force. 


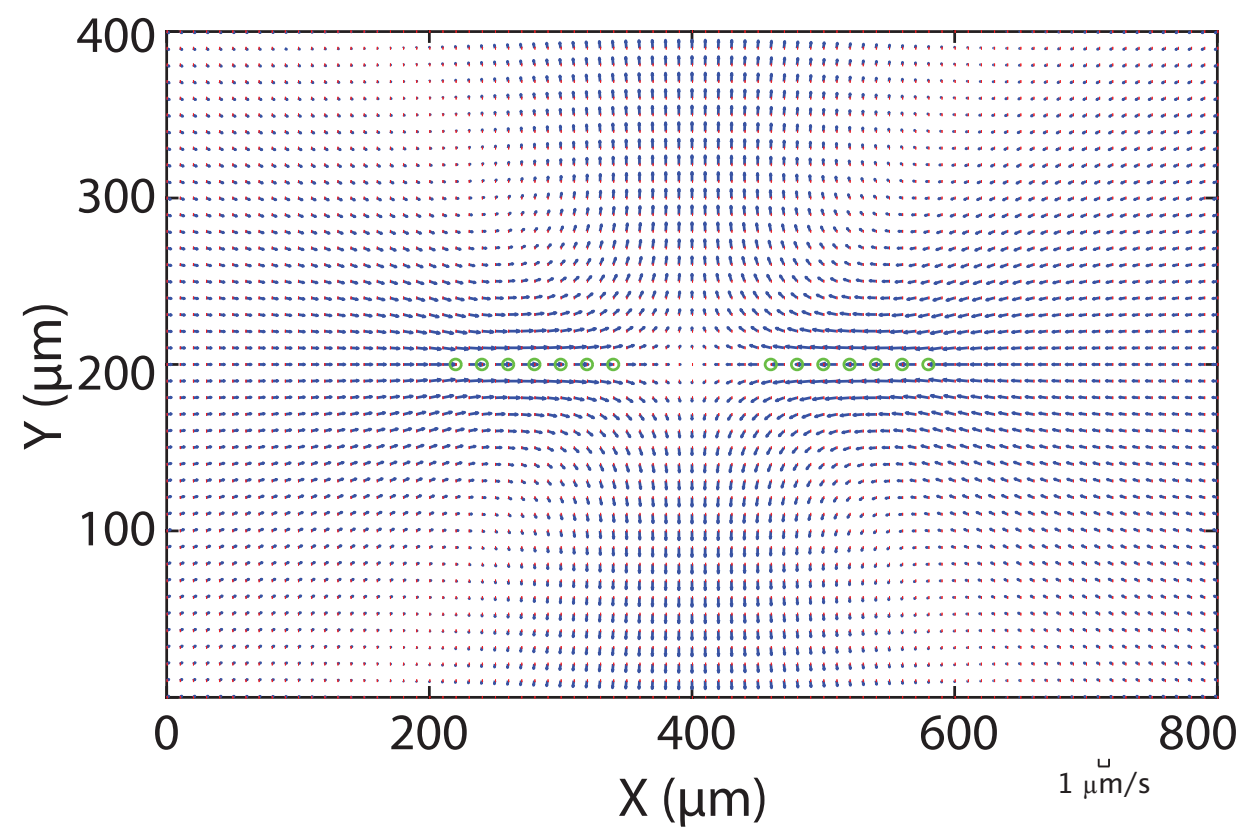

Figure S12: Flow field generated by 14 Stokeslets, indicated by green circles, to model the 350 $\mu \mathrm{m}$ activation bar case. This theoretical model recovers the inflows and outflows observed in the experiment (Fig. 4A), but not the vortices.

Due to the linear nature of low-Reynolds-number flow [39], we expect that the velocity field generated by a complex light pattern can be retrieved by superposition of simple patterns. To confirm this, we superimpose flow fields from single bars to mimic the flow field generated by "L", "+" and "T"-shaped light patterns (Fig. S13). For the "+" case, the superimposed fields closely resembles the experimentally observed field (Fig. S13C). The "L" and "T"-shaped cases are roughly similar to the experimental results, but direction of the inflows do not match (Fig. S13B, D). 

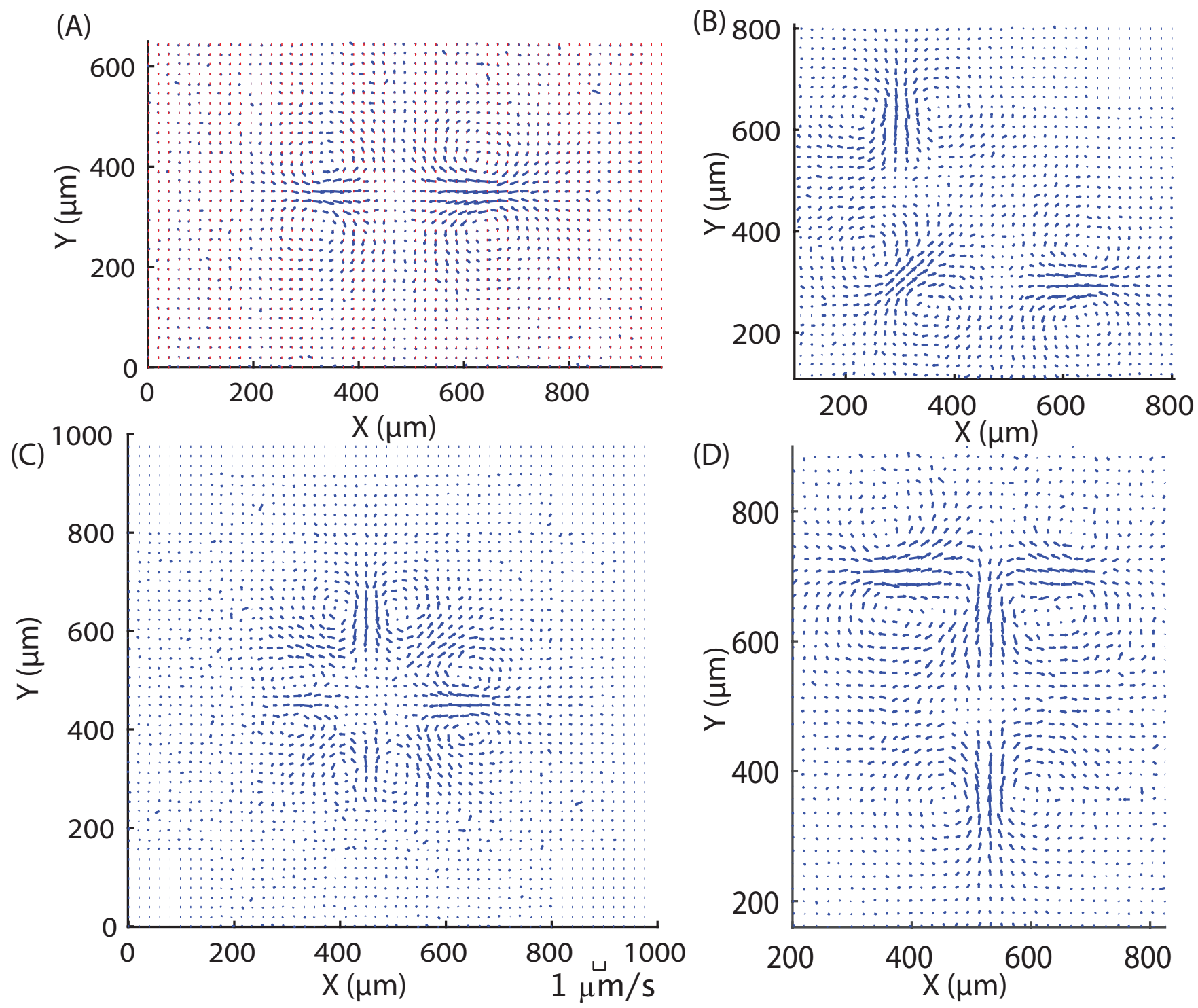

Figure S13: Demonstration of the linearity of the flow field. (A) A time averaged flow field generated by a $350 \mu \mathrm{m}$ rectangular bar. Flow fields generated by the rotation and superposition of the flow field in (A) to retrieve flow fields for (B)"L", (C) "+", and (D) "T"-shaped light patterns.

To model the "L" and "T" flow fields more accurately, we generate the flow field for a series of Stokeslets following the geometry of the microtubule structure, rather than the light pattern itself. Using this method, the modeled flow fields are a good approximation of the observed flow fields. The inflows and outflows match the experimentally observed positions and orientations (Fig. S14). This result implies that the observed flow patterns are set by the microtubule structure rather than the light pattern. 

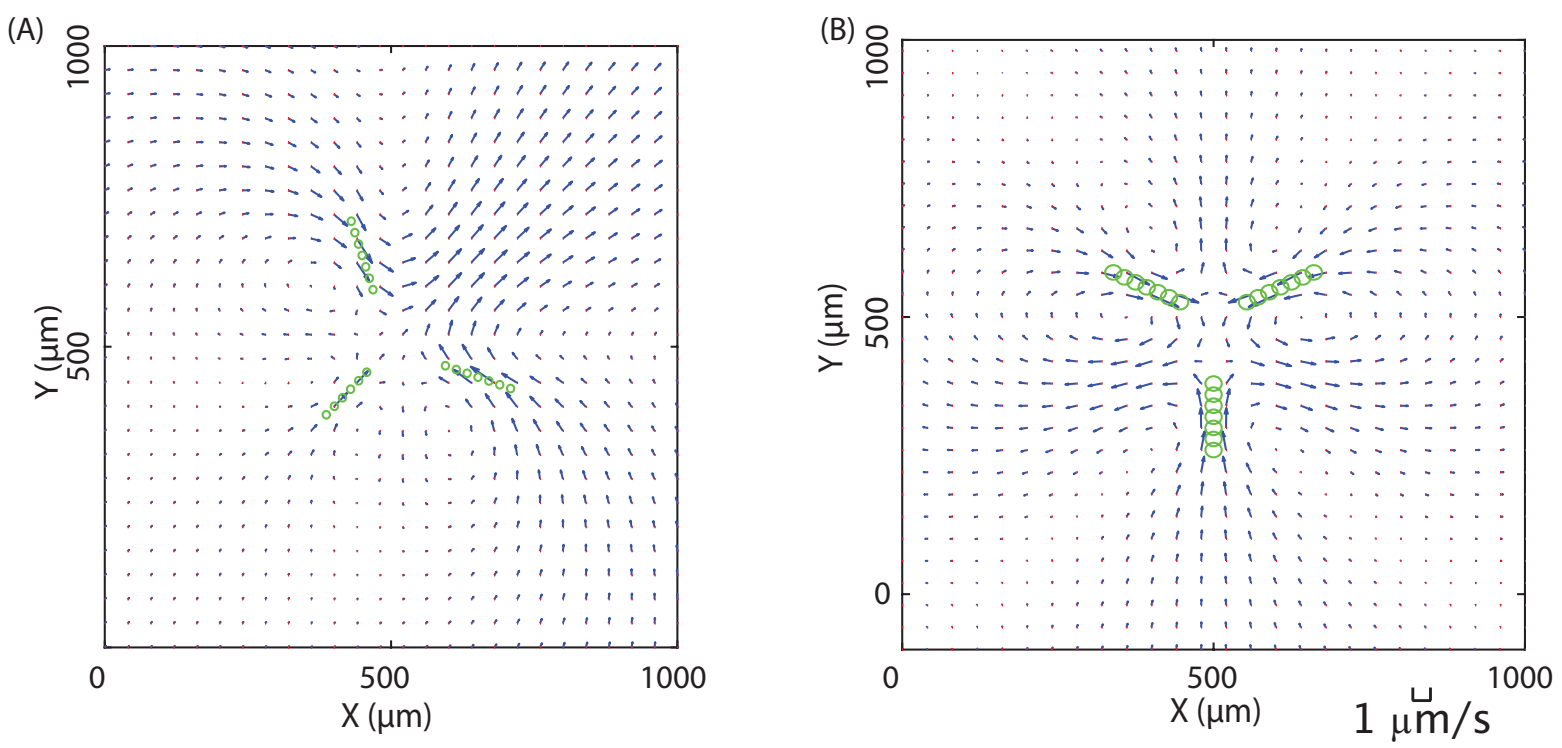

Figure S14: Theoretical simulation of fluid flows under complex light patterns using Stokeslets. The Stokeslets are positioned following the shape of the microtuble network observed in Fig. 4F. Green circles denote the Stokeslets. (A) Flow field for "L"-shaped light pattern. (B) Flow field for "T"-shaped light pattern.

\section{References}

1. Marchetti, M. C. et al. Hydrodynamics of soft active matter. Rev. Mod. Phys. 85, 1143-1189 (3 July 2013).

2. Dumont, S. \& Prakash, M. Emergent mechanics of biological structures. Molecular Biology of the Cell 25, 3461-3465. ISSN: 1059-1524, 1939-4586 (Nov. 5, 2014).

3. Needleman, D. \& Dogic, Z. Active matter at the interface between materials science and cell biology. Nature Reviews Materials 2, 17048. ISSN: 2058-8437 (Sept. 2017).

4. Nédélec, F. J., Surrey, T., Maggs, A. C. \& Leibler, S. Self-organization of microtubules and motors. Nature 389, 305. ISSN: 1476-4687 (Sept. 1997).

5. Surrey, T., Nédélec, F., Leibler, S. \& Karsenti, E. Physical Properties Determining SelfOrganization of Motors and Microtubules. Science 292, 1167-1171. ISSN: 0036-8075, 1095-9203 (May 11, 2001).

6. Sanchez, T., Chen, D. T. N., DeCamp, S. J., Heymann, M. \& Dogic, Z. Spontaneous motion in hierarchically assembled active matter. Nature 491, 431. ISSN: 1476-4687 (Nov. 2012).

7. DeCamp, S. J., Redner, G. S., Baskaran, A., Hagan, M. F. \& Dogic, Z. Orientational order of motile defects in active nematics. Nature Materials 14, 1110. ISSN: 1476-4660 (Nov. 2015).

8. Wu, K.-T. et al. Transition from turbulent to coherent flows in confined three-dimensional active fluids. Science 355, eaal1979. ISSN: 0036-8075, 1095-9203 (Mar. 24, 2017).

9. Bricard, A., Caussin, J.-B., Desreumaux, N., Dauchot, O. \& Bartolo, D. Emergence of macroscopic directed motion in populations of motile colloids. Nature 503, 95-98. ISSN: 1476-4687 (Nov. 2013). 
10. Palacci, J., Sacanna, S., Steinberg, A. P., Pine, D. J. \& Chaikin, P. M. Living Crystals of Light-Activated Colloidal Surfers. Science 339, 936-940. ISSN: 0036-8075, 1095-9203 (Feb. 22, 2013).

11. Keya, J. J. et al. DNA-assisted swarm control in a biomolecular motor system. Nature Communications 9, 453. ISSN: 2041-1723 (Jan. 2018).

12. Aoyama, S., Shimoike, M. \& Hiratsuka, Y. Self-organized optical device driven by motor proteins. Proceedings of the National Academy of Sciences 110, 16408-16413. ISSN: 0027-8424, 1091-6490 (Oct. 8, 2013).

13. Tas, R. P. et al. Guided by Light: Optical Control of Microtubule Gliding Assays. Nano Letters 0. PMID: 30449112, null (0).

14. Fletcher, D. A. \& Mullins, R. D. Cell mechanics and the cytoskeleton. Nature 463, 485-492. ISSN: 0028-0836 (Jan. 28, 2010).

15. Nédélec, F., Surrey, T. \& Maggs, A. C. Dynamic Concentration of Motors in Microtubule Arrays. Physical Review Letters 86, 3192-3195 (Apr. 2, 2001).

16. Lee, H. Y. \& Kardar, M. Macroscopic equations for pattern formation in mixtures of microtubules and molecular motors. Physical Review. E, Statistical, Nonlinear, and Soft Matter Physics 64, 056113. ISSN: 1539-3755 (Nov. 2001).

17. Keber, F. C. et al. Topology and dynamics of active nematic vesicles. Science 345, 1135-1139. ISSN: 0036-8075, 1095-9203 (Sept. 5, 2014).

18. Schuppler, M., Keber, F. C., Kröger, M. \& Bausch, A. R. Boundaries steer the contraction of active gels. Nature Communications 7, 13120. ISSN: 2041-1723 (Oct. 14, 2016).

19. Good, M. C., Vahey, M. D., Skandarajah, A., Fletcher, D. A. \& Heald, R. Cytoplasmic Volume Modulates Spindle Size During Embryogenesis. Science 342, 856-860. ISSN: 0036-8075, 10959203 (Nov. 15, 2013).

20. Gardel, M. L., Schneider, I. C., Aratyn-Schaus, Y. \& Waterman, C. M. Mechanical Integration of Actin and Adhesion Dynamics in Cell Migration. Annual Review of Cell and Developmental Biology 26, 315-333 (2010).

21. Theurkauf, W. E. Premature microtubule-dependent cytoplasmic streaming in cappuccino and spire mutant oocytes. Science 265, 2093-2096 (1994).

22. Woodhouse, F. G. \& Goldstein, R. E. Cytoplasmic streaming in plant cells emerges naturally by microfilament self-organization. Proceedings of the National Academy of Sciences 110, 1413214137 (2013).

23. Ganguly, S., Williams, L. S., Palacios, I. M. \& Goldstein, R. E. Cytoplasmic streaming in Drosophila oocytes varies with kinesin activity and correlates with the microtubule cytoskeleton architecture. Proceedings of the National Academy of Sciences 109, 15109-15114 (2012).

24. Goldstein, R. E., Tuval, I. \& van de Meent, J.-W. Microfluidics of cytoplasmic streaming and its implications for intracellular transport. Proceedings of the National Academy of Sciences 105, 3663-3667 (2008).

25. Drescher, K., Dunkel, J., Cisneros, L. H., Ganguly, S. \& Goldstein, R. E. Fluid dynamics and noise in bacterial cell-cell and cell-surface scattering. Proceedings of the National Academy of Sciences 108, 10940-10945 (2011).

26. Drescher, K., Goldstein, R. E., Michel, N., Polin, M. \& Tuval, I. Direct Measurement of the Flow Field around Swimming Microorganisms. Phys. Rev. Lett. 105, 168101 (16 Oct. 2010).

27. Guntas, G. et al. Engineering an improved light-induced dimer (iLID) for controlling the localization and activity of signaling proteins. Proceedings of the National Academy of Sciences of the United States of America 112, 112-117. ISSN: 1091-6490 (Jan. 6, 2015). 
28. https://mitchison.hms.harvard.edu/files/mitchisonlab/files/tubulin_polymerization_with_ gtp.pdf.

29. Lau, A. W. C., Prasad, A. \& Dogic, Z. Condensation of isolated semi-flexible filaments driven by depletion interactions. EPL (Europhysics Letters) 87, 48006 (2009).

30. Edelstein, A., Amodaj, N., Hoover, K., Vale, R. \& Stuurman, N. Computer Control of Microscopes Using $\mu$ Manager. Current Protocols in Molecular Biology 92, 14.20.1-14.20.17 (2010).

31. Tirado, M. M. \& Garcia de la Torre, G. Rotational Dynamics of Rigid, Symmetric Top Macomolecules: Application to circular cylinders. Journal of Chemical Physics 73, 1986-1993 (1980).

32. Svoboda, K. \& Block, S. M. Biological Applications of Optical Forces. Annual Review of Biophysics and Biomolecular Structure 23, 247-285 (1994).

33. Maas, H., Gruen, A. \& Papantoniou, D. Particle tracking velocimetry in three-dimensional flows. Experiments in Fluids 15, 133-146 (1993).

34. Schmidt, T., Schütz, G., Baumgartner, W., Gruber, H. \& Schindler, H. Imaging of single molecule diffusion. Proceedings of the National Academy of Sciences 93, 2926-2929 (1996).

35. Panton, R. L. Incompressible flow (John Wiley \& Sons, 2006).

36. Dunkel, J. et al. Fluid dynamics of bacterial turbulence. Physical review letters 110, 228102 (2013).

37. Happel, J. \& Brenner, H. Low Reynolds number hydrodynamics: with special applications to particulate media (Springer Science \& Business Media, 2012).

38. Chwang, A. T. \& Wu, T. Y.-T. Hydromechanics of low-Reynolds-number flow. Part 2. Singularity method for Stokes flows. Journal of Fluid Mechanics 67, 787-815 (1975).

39. Kim, S. \& Karrila, S. J. Microhydrodynamics: principles and selected applications (Courier Corporation, 2013). 